\title{
Heats, Equilibrium Constants, and Free Energies of Formation of the $\mathrm{C}_{3}$ to $\mathrm{C}_{5}$ Diolefins, Styrene, and the Methylstyrenes ${ }^{1}$
}

\author{
By John E. Kilpatrick, ${ }^{2}$ Charles W. Beckett, ${ }^{3}$ Edward J. Prosen, Kenneth S. Pitzer, ${ }^{4}$ \\ and Frederick D. Rossini ${ }^{5}$
}

\begin{abstract}
For the $\mathrm{C}_{3}$ to $\mathrm{C}_{5}$ diolefins, styrene, and the methylstyrenes, values are presented for the following thermodynamic properties to $1,500^{\circ} \mathrm{K}$ : Heat-content function, free-energy function, entropy, heat content, heat capacity, heat of formation from the elements, free energy of formation from the elements, and logarithm of the equilibrium constant of formation from the elements. Equilibrium constants and concentrations are given in tabular and graphical form for some reactions of isomerization and dehydrogenation.
\end{abstract}

\section{Introduction}

As part of the work of the American Petroleum Institute Research Project 44 at the National Bureau of Standards and the University of California, values have been compiled for the thermodynamic properties, in the gaseous state to $1,500^{\circ}$ $\mathrm{K}$, of the heat-content function, free-energy function, entropy, heat content, heat capacity, heat of formation, free energy of formation, and logarithm of the equilibrium constant of formation for the $\mathrm{C}_{3}$ to $\mathrm{C}_{5}$ diolefins, styrene, and the methylstyrenes. Calculations have also been made of the free energies and equilibrium constants of a number of reactions involving isomerization and hydrogenation of these compounds.

\section{Constants}

The values of the constants used in the present calculations are as follows $[1,2]:{ }^{6}$ The calorie

\footnotetext{
1 This investigation was performed as a part of the work of the American Petroleum Institute Research Project 44 at the National Bureau of Standards and the University of California.

${ }^{2}$ Research Associate on the American Petroleum Institute Research Project 44 at the National Bureau of Standards.

${ }^{3}$ Research Associate on the American Petroleum Institute Research Project 44 at the University of California.

${ }^{4}$ Associate Director of the American Petroleum Institute Research Project 44; Professor Chemistry at the University of California, Berkeley, California.

${ }^{5}$ Director of the American Petroleum Institute Research Project 44 at the National Bureau of Standards.

${ }^{6}$ Figures in brackets indicate the literature references at the end of this paper.
}

used is the conventional thermochemical calorie defined as 4.1840 absolute joules; the absolute temperature of the ice point is $273.160 \pm 0.010^{\circ} \mathrm{K}$; the value of the radiation constant, $h c / k$, is 1.43847 $\pm 0.00045 \mathrm{~cm} \mathrm{deg}$; the gas constant is 1.98719 $\pm 0.00013 \mathrm{cal} / \mathrm{deg}$ mole. Values of other constants related to these are given in references $[1,2]$.

\section{Heat-Content Function, Free-Energy Function, Entropy, Heat Content, and Heat Capacity}

\section{1. $\mathrm{C}_{3}$ to $\mathrm{C}_{5}$ Diolefins}

For propadiene (allene), the thermodynamic functions were computed by using the following: The assignment of frequencies given by Thompson and Harris [3], which differs slightly from previous assignments $[4,5]$, two equal moments of inertia $\left(9.82 \times 10^{-39} \mathrm{~g} \mathrm{~cm}^{2}\right)$ recalculated from the spectral data on the rotational fine structure [6], a third moment of inertia $\left(0.575 \times 10^{-39} \mathrm{~g} \mathrm{~cm}^{2}\right)$ assumed to be equal to that of ethylene [7], and a symmetry number of four. The foregoing moments of inertia correspond to the following dimensions of the molecule of propadiene, which agree well with those obtained from measurements of electron diffraction [8]: $\mathrm{C}=\mathrm{C}, 1.34 \mathrm{~A} ; \mathrm{C}-\mathrm{H}, 1.07 \mathrm{~A}$; $\mathrm{H}-\mathrm{C}-\mathrm{H}$ angle, $120^{\circ}$. The uncertainty in the 
calculated values of the thermodynamic functions is estimated to be about $0.2 \mathrm{cal} / \mathrm{deg}$ mole at the lower temperatures and somewhat greater at the higher temperatures because of the neglect of vibrational anharmonicities. The values of heat capacity calculated for propadiene in the present investigation agree very well with the experimental data that have been reported $[4,9,10]$. Some values of entropy previously calculated for propadiene by other workers [11, 12], however, differ markedly, for some unknown cause, from those given in the present report.

For 1,2-butadiene, the thermodynamic functions were computed by using the following: The assignment of frequencies given by Szasz, McCartney, and Rank [13]; moments of inertia based on the same corresponding dimensions as for propadiene, together with additional dimensions of $1.54 \mathrm{~A}$ for $\mathrm{C}-\mathrm{C}$ and $1.09 \mathrm{~A}$ for $\mathrm{H}-\mathrm{CCH}_{3}$, giving $I_{1}=2.819 \times 10^{-39}, I_{2}=20.31 \times 10^{-39}$, and $I_{3}=22.03$ $\times 10^{-39} \mathrm{~g} \mathrm{~cm}^{2}$; a reduced moment of inertia for the methyl rotation of $4.78 \times 10^{-40} \mathrm{~g} \mathrm{~cm}^{2}$ corresponding to $I_{\mathrm{CH}_{3}}=5.30 \times 10^{-40} \mathrm{~g} \mathrm{~cm}^{2}$; and a potential barrier restricting internal rotation of $1,800 \mathrm{cal} / \mathrm{mole}$, which was selected to fit the experimental value of entropy of gaseous 1,2-butadiene at $273.25^{\circ} \mathrm{K}$ reported by Aston and Szasz [14], including a calorimetrically measured entropy of vaporization. The potential barrier from the present calculations is slightly different from that reported by Aston and Szasz [14] because of the difference in the molecular dimensions used in the respective calculations. As might be expected, the potential barrier for 1,2-butadiene is about the same as that for propylene. The assignment of vibrational frequencies made by Szasz, McCartney, and Rank [13] was tested by calculating approximate product ratios for methyl substitutions of the type used by Pitzer and Scott [15], in which calculations the values of the product ratios for 1,2-butadiene agree very well with those for the methylbenzenes. This agreement offers considerable confirmation of the assignment of frequencies for 1,2-butadiens.

For 1,3-butadiene, the available spectroscopic and other molecular data appear to be satisfactorily represented by the values of the thermodynamic functions calculated by Aston, Szasz, Wooley, and Brickwedde [16], and their values for the heat-content function, free-energy function, and heat capacity were used in the present calculations. However, it should be noted that the available spectroscopic and other molecular data are not sufficient to confirm the correctness of the details of their molecular model of 1,3butadiene.

For the pentadienes, the available thermal, spectroscopic, and other molecular data are either insufficient or too uncertain to permit a satisfactory statistical treatment at the present time. However, by taking advantage of regularities in the properties of related compounds, thermodynamic functions for the pentadienes may be calculated by suitable combination of the thermodynamic functions for other compounds for which the appropriate values have already been calculated [15, 17, 18, 19]. For such calculations by the method of increments, the following equations were used to evaluate the thermodynamic functions for the seven pentadienes:

$$
\begin{gathered}
G(1,2 \text {-pentadiene })=G(1 \text {-butene })+ \\
G(1,2 \text {-butadiene })-G(\text { propylene }) .
\end{gathered}
$$

$$
\begin{gathered}
G(2,3 \text {-pentadiene })=2 G(1,2 \text {-butadiene })- \\
G(\text { propadiene })-R \text { l } 8 .
\end{gathered}
$$

$G(3$-methyl-1,2-butadiene $)=G($ isobutene $)+(3)$ $G(1,2$-butadiene)- $G$ (propylene).

$G(c i s-1,3$-pentadiene $)=G($ cis-2-butene $)+$

$$
G(1,3 \text {-butadiene })-G \text { (propylene) }+R \ln 4 \text {. }
$$

$G($ trans-1,3-pentadiene $)=G($ trans-2-butene $)+$ $G(1,3$-butadiene $)-G$ (propylene) $+R \ln 4$.

$G(2$-methyl-1,3-butadiene $)=G($ isobutene $)+$ $G(1,3$-butadiene $)-G($ propylene $+R \ln 4-0.3$.

$G(1,4$-pentadiene $)=2 G(1$-butene $)-$

$$
G \text { (propane)-R } \ln 4 \text {. }
$$

In the foregoing equations, $G$ represents the heat content function, the negative of the free energy function, or the heat capacity. The constant terms, $R \ln 8$, etc., appearing, respectively, in eq 2 , $4,5,6$, and 7 , are corrections for the symmetry number and are to be used for the negative of the free-energy function, $-\left(F^{\circ}-H_{0}^{\circ}\right) / T$, but not for the heat-content function or the heat capacity. The values of the thermodynamic functions used for the calculations represented by eq 1 to 7 , inclusive, are from the following: Propane [18]; propylene, 1-butene, cis-2-butene, trans-2-butene, and isobutene [19]. 
There are now available a number of checks on the accuracy of the increment method for calculating thermodynamic functions, as illustrated by eq 1 to 7 , inclusive. Some confirmation of the increment method has already been reported for certain alkylbenzenes $[15,20]$ and for certain alkylcyclohexanes [17, 21].

In 1946, values of the thermodynamic functions for 2-methyl-2-butene were calculated [22] by means of the relation

$G(2$-methyl-2-butene $)=2 / 3 G($ cis-2-butene + trans 2 -butene + isobutene $)-G$ (propylene $)+R \ln 4$.

This yielded $80.90 \mathrm{cal} / \mathrm{deg}$ mole for the standard entropy in the gaseous state at $25^{\circ} \mathrm{C}$, which is to be compared with the value 80.92 subsequently available from the experimental work of Todd, Oliver, and Huffman [22], and Huffman and Scott [23]. The calculated value for the heat capacity of gaseous 2-methyl-2-butene is about 1 percent higher than the experiment value that has recently become available [23].

Before the recent experimental work on 1,2butadiene became available [14], the authors had calculated the thermodynamic functions of 1,2butadiene by the method of increments with what now appear to be excellent results, using the equation

$$
\begin{aligned}
& G(1,2 \text {-butadiene })=G(\text { allene })+G(\text { propylene })- \\
& G(\text { ethylene }) .
\end{aligned}
$$

The standard entropy of gaseous 1,2-butadiene at $25^{\circ} \mathrm{C}$ was calculated by the method of increments to be $69.7 \mathrm{cal} / \mathrm{deg}$ mole, which is to be compared with the new calculated value of $70.03 \pm 0.20$ cal/deg mole.

There is an experimental entropy available for comparison with our result for 2-methyl-1,3butadiene (isoprene). The experimental value, $75.2 \pm 0.3,[24,25]$ is $0.5 \mathrm{cal} / \mathrm{deg}$ mole lower than the value given by the increment equation without the term -0.3. Although this difference is well within the expected uncertainties of the method, the additional constant term $(-0.3)$ was added to the increment equation, in order to have better agreement with the isoprene experimental value at $298.16^{\circ} \mathrm{K}$. Presumably, this change gives better extrapolated values at higher temperatures.

In connection with the calculation of the thermodynamic functions of the pentadienes by the method of increments, it is estimated that the over-all uncertainty is about $1 \mathrm{cal} / \mathrm{deg}$ mole at room temperature and greater at higher temperatures, the values for 1,4-pentadiene being particularly uncertain. However, the differences between values of the thermodynamic functions at given temperatures are more significant than the over-all uncertainties.

The resulting values of the thermodynamic functions for the diolefins, $\mathrm{C}_{3}$ to $\mathrm{C}_{5}$, are given in tables 1 to 5 , inclusive, which give values of the following properties, respectively, from $0^{\circ}$ to $1,500^{\circ} \mathrm{K}$ : Heat-content function, free-energy function, entropy, heat content, and heat capacity. The value of the entropy is obtained as the value of the heat-content function less the value of the free-energy function, and the value of the heat content is obtained by multiplying the value of the heat-content function by the temperature.

\begin{tabular}{|c|c|c|c|c|c|c|c|c|c|c|c|c|c|c|c|c|}
\hline \multirow{3}{*}{$\begin{array}{l}\text { Compound } \\
\text { (gas) }\end{array}$} & \multirow{3}{*}{$\begin{array}{l}\text { For- } \\
\text { mula }\end{array}$} & \multicolumn{15}{|c|}{ Temperature in ${ }^{\circ} \mathrm{K}$} \\
\hline & & 0 & 298.16 & 300 & 400 & 500 & 600 & 700 & 800 & 900 & 1,000 & 1,100 & 1,200 & 1,300 & 1,400 & 1,500 \\
\hline & & \multicolumn{15}{|c|}{ Heat-content function, $\left(H^{\circ}-H_{0}^{\circ}\right) / T$, in cal/deg mole } \\
\hline Propadiene (allene) & $\mathrm{C}_{3} \mathrm{H}_{4}$ & 0 & 10.12 & 10.15 & 11.54 & 12.94 & 14.28 & 15.51 & 16.66 & 17. 71 & 18.68 & 19.57 & 20.40 & 21.17 & 21.88 & 22.54 \\
\hline 1,2-Butadiene & $\mathrm{C}_{4} \mathrm{H}_{6}$ & 0 & 12.92 & 12. 96 & 15.07 & 17.16 & 19.15 & 21.01 & 22.74 & 24.34 & 25.82 & 27.18 & 28.44 & 29.62 & 30.70 & 31.70 \\
\hline 1,3-Butadiene. . . . & $\mathrm{C}_{4} \mathrm{H}_{6}$ & 0 & 12.16 & 12.20 & 14.59 & 16.97 & 19. 18 & 21.19 & 23.01 & 24.66 & 26.16 & 27.54 & 28.80 & 29.96 & 31.03 & 32.02 \\
\hline 1,2-Péntadiene $\ldots \ldots \ldots$ & $\mathrm{C}_{5} \mathrm{H}_{8}$ & 0 & 16. 2 & 16.3 & 19.3 & 22.3 & 25.0 & 27.6 & 29.9 & 32.0 & 34.0 & 35.8 & 37.4 & 39.0 & 40.4 & 41.7 \\
\hline cis-1,3-Pentadiene (cis-piperylene) ..... & $\dot{\mathrm{C}}_{5} \mathrm{H}_{8}$ & 0 & 14.5 & 14.6 & 17.5 & 20.5 & 23.4 & 26.0 & 28.5 & 30.7 & 32.7 & 34.6 & 36.3 & 37.9 & 39.4 & 40.7 \\
\hline trans-1,3-Pentadiene (trans-piperylene) & $\mathrm{C}_{5} \mathrm{H}_{8}$ & 0 & 15.3 & 15.4 & 18.6 & 21.7 & 24.5 & 27.1 & 29.5 & 31.7 & 33.7 & 35.5 & 37.2 & 38.7 & 40.1 & 41.4 \\
\hline 1,4-Pentadiene $\ldots$ & $\mathrm{C}_{5} \mathrm{H}_{8}$ & 0 & 16.5 & 16.6 & 19.6 & 22.4 & 25.2 & 27.7 & 29.9 & 32.0 & 34.0 & 35.8 & 37.4 & 39.0 & 40.4 & 41.7 \\
\hline 2,3-Pentadiene............. & $\mathrm{C}_{5} \mathrm{H}_{8}$ & 0 & 15.7 & 15.8 & 18.6 & 21.4 & 24.0 & 26.5 & 28.8 & 31.0 & 33.0 & 34.8 & 36.5 & 38.1 & 39.5 & 40.9 \\
\hline 3-Methyl-1,2-butadiene.. & $\mathrm{C}_{5} \mathrm{H}_{8}$ & 0 & 15.8 & 15.8 & 18.9 & 21.8 & 24.6 & 27.1 & 29.4 & 31.5 & 33.5 & 35.3 & 37.0 & 38.6 & 40.0 & 41.3 \\
\hline 2-Methyl-1,3-butadiene (isoprene) ..... & $\mathrm{C}_{5} \mathrm{H}_{8}$ & 0 & 15. 0 & 15.1 & 18.4 & 21.7 & 24.6 & 27.3 & 29.7 & 31.9 & 33.8 & 35.7 & 37.4 & 38.9 & 40.3 & 41.6 \\
\hline
\end{tabular}

TABLE 1. Values of the heat-content function, for the ideal gaseous state, to $1,500^{\circ} \mathrm{K}$, for the $\mathrm{C}_{3}$ to $\mathbf{C}_{5}$ diolefins 
TABLE 2. Values of the free-energy function, for the ideal gaseous state, to $1,500^{\circ} \mathrm{K}$, for the $\mathrm{C}_{3}$ to $\mathrm{C}_{5}$ diolefins

\begin{tabular}{|c|c|c|c|c|c|c|c|c|c|c|c|c|c|c|c|c|}
\hline \multirow{3}{*}{ Compound (gas) } & \multirow{3}{*}{$\begin{array}{l}\text { For- } \\
\text { mula }\end{array}$} & \multicolumn{15}{|c|}{ Temperature in ${ }^{\circ} \mathrm{K}$} \\
\hline & & 0 & 298.16 & 300 & 400 & 500 & 600 & 700 & 800 & 900 & 1,000 & 1,100 & 1,200 & 1,300 & 1,400 & 1,500 \\
\hline & & \multicolumn{15}{|c|}{ Free-energy function, $\left(F^{\circ}-H_{0}^{\circ}\right) / T$, in cal $/$ deg mole } \\
\hline Propadiene (allene) & $\mathrm{C}_{3} \mathrm{H}_{4}$ & & -48.18 & -48.24 & -51.35 & -54.08 & -56.55 & -58.85 & -61.00 & -63.02 & -64.94 & -66.76 & -68.50 & -70.16 & -71.76 & -73.29 \\
\hline 1,2 -Butadiene $\ldots . .$. & $\mathrm{C}_{4} \mathrm{H}_{6}$ & 0 & -57.11 & -57.19 & -61.21 & -64.79 & -68.10 & -71.19 & -74.11 & -76.88 & -79.52 & -82.04 & -84.47 & -86.80 & -89.02 & -91.18 \\
\hline cis-1,3-Pentadiene (cis-piperylene) & $\mathrm{C}_{5} \mathrm{H}_{8}$ & & -62.9 & -63.0 & -67.6 & -71.8 & -75.8 & -79.6 & -83.3 & -86.7 & -90.1 & -93.3 & -96.4 & -99.3 & $|-102.2|$ & -105.0 \\
\hline $\begin{array}{l}\text { trans-1,3-Pentadiene } \quad \text { (trans-pip- } \\
\text { erylene) }\end{array}$ & $\mathrm{C}_{5} \mathrm{H}_{8}$ & & -61.1 & -61.2 & -66.1 & -70.5 & -74.7 & -78.7 & -82.5 & -86.1 & -89.5 & -92.8 & -96.0 & -99.0 & -101.9 & -104.8 \\
\hline 1,4-Pentadiene. & $\mathrm{C}_{5} \mathrm{H}_{8}$ & & -63.2 & -63.3 & -68.5 & -73.1 & -77.4 & -81.5 & -85.4 & -89.0 & -92.5 & -95.8 & -99.0 & -102.0 & -105.0 & -107.8 \\
\hline 2,3-Pentadiene & $\mathrm{C}_{5} \mathrm{H}_{8}$ & & -61.9 & -62.0 & -66.9 & -71.4 & -75.5 & -79.4 & -83.1 & -86.6 & -90.0 & -93.2 & -96.3 & -99.3 & -102.2 & -104.9 \\
\hline
\end{tabular}

TABLE 3. Values of the entropy, for the ideal gaseous state, to $1,500^{\circ} \mathrm{K}$, for the $\mathrm{C}_{3}$ to $\mathrm{C}_{5}$ diolefins

\begin{tabular}{|c|c|c|c|c|c|c|c|c|c|c|c|c|c|c|c|c|}
\hline \multirow{3}{*}{ Compound (gas) } & \multirow{3}{*}{$\begin{array}{c}\text { Form- } \\
\text { ula }\end{array}$} & \multicolumn{15}{|c|}{ Temperature in ${ }^{\circ} \mathbf{K}$} \\
\hline & & 0 & 298.16 & 300 & 400 & 500 & 600 & 700 & 800 & 900 & 1,000 & 1,100 & 1,200 & 1,300 & 1,400 & 1,500 \\
\hline & & \multicolumn{15}{|c|}{ Entropy, $S^{\circ}$, in cal/deg mole } \\
\hline Propadiene (allene). & $\mathrm{C}_{3} \mathrm{H}_{4}$ & 0 & 58.30 & 58.39 & 62.89 & 67.02 & 70.83 & 74.36 & 77.65 & 80.73 & 83.61 & 86. 33 & 88.90 & 91.33 & 93.64 & 95.83 \\
\hline 1,2-Butadiene. ...... & $\mathrm{C}_{4} \mathrm{H}_{6}$ & 0 & 70.03 & 70.15 & 76.28 & 81.95 & 87.25 & 92.20 & 96.85 & 101.22 & 105.34 & 109. 22 & 112.91 & 116.42 & 119. 72 & 122.88 \\
\hline 1,3-Butadiene ...... & $\mathrm{C}_{4} \mathrm{H}_{6}$ & 0 & 66.62 & 66.74 & 72.97 & 78.86 & 84.36 & 89.48 & 94.25 & 98.71 & 102.88 & 106.82 & 110.53 & 114.05 & 117.38 & 120.54 \\
\hline 1,2-Pentadiene $\ldots . . . .$. & $\mathrm{C}_{5} \mathrm{H}_{8}$ & 0 & 79.7 & 79.9 & $88.0+$ & 95.6 & 102.6 & 109.3 & 115.4 & 121.1 & 126.6 & 131.7 & 136.5 & 141.2 & 145.5 & 149. 7 \\
\hline cis-1,3-Pentadiene (cis-piperylene) ..... & $\mathrm{C}_{5} \mathrm{H}_{8}$ & 0 & 77.5 & 77.6 & 85.1 & 92.3 & 99.2 & 105. 7 & 111.7 & 117.4 & 122.18 & 127.9 & 132.7 & 137.2 & 141.6 & 145. 7 \\
\hline $\begin{array}{l}\text { trans-1,3-Pentadiene } \quad \text { (trans-piper- } \\
\text { ylene) }\end{array}$ & $\mathrm{C}_{5} \mathrm{H}_{8}$ & 0 & 76.4 & 76.8 & 84.6 & 92.2 & 99.2 & 105.8 & 112.0 & 117.8 & 123.2 & 128.3 & 133.1 & 137.7 & 142.1 & 146. 2 \\
\hline 1,4-Pentadiene. & $\mathrm{C}_{5} \mathrm{H}_{8}$ & 0 & 79.7 & 79.9 & 88.1 & 95.5 & 102.6 & 109. 2 & 115.3 & 121.0 & 126.5 & 131.6 & 136.4 & 141.0 & 145.3 & 149.5 \\
\hline 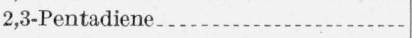 & $\mathrm{C}_{5} \mathrm{H}_{8}$ & 0 & 77.6 & 77.8 & 85.5 & 92.8 & 99.5 & 105.9 & 111.9 & 117.6 & 123.0 & 128.0 & 132.8 & 137.4 & 141. 7 & 145.8 \\
\hline 3-Methyl-1,2-butadiene.... & $\mathrm{C}_{5} \mathrm{H}_{8}$ & 0 & 76.4 & 76.5 & 84.6 & 92.0 & 99.1 & 105.6 & 111.6 & 117.3 & 122.7 & 127. 8 & 132.7 & 137.3 & 141.6 & 145.7 \\
\hline 2-Methyl-1,3-butadiene (isoprene) ..... & $\mathrm{C}_{5} \mathrm{H}_{8}$ & 0 & 75.4 & 75.6 & 83.7 & 91.4 & 98.6 & 105.3 & 111.5 & 117.3 & 122.7 & 127.9 & 132.7 & 137.3 & 141.7 & 145.8 \\
\hline
\end{tabular}

TABLE 4. Values of the heat content, for the ideal gaseous state, to $1,500^{\circ} \mathrm{K}$, for the $\mathrm{C}_{3}$ to $\mathrm{C}_{5}$ diolefins

\begin{tabular}{|c|c|c|c|c|c|c|c|c|c|c|c|c|c|c|c|c|}
\hline \multirow{3}{*}{ Compound (gas) } & \multirow{3}{*}{$\begin{array}{l}\text { For- } \\
\text { mula }\end{array}$} & \multicolumn{15}{|c|}{ Temperature in ${ }^{\circ} \mathrm{K}$} \\
\hline & & 0 & 298.16 & 300 & 400 & 500 & 600 & 700 & 800 & 900 & 1,000 & 1,100 & 1,200 & 1,300 & 1,400 & 1,500 \\
\hline & & \multicolumn{15}{|c|}{ Heat content, $\left(H^{\circ}-H_{0}^{\circ}\right)$, in cal $/$ mole } \\
\hline Propadiene (allene). & $\mathrm{C}_{3} \mathrm{H}_{4}$ & 0 & 3017.4 & 3045.0 & 4616.0 & 6470.0 & 8568.0 & 10857.0 & 13328.0 & 15939.0 & 18680.0 & 21527.0 & 24480.0 & 27521.0 & 30632.0 & 33810.0 \\
\hline 1,2-Butadiene...... & $\mathrm{C}_{4} \mathrm{H}_{6}$ & 0 & 3852.2 & 3888.0 & 6028.0 & 8580.0 & 11490.0 & 14707.0 & 18192.0 & 21906.0 & 25820.0 & 29898.0 & 34128.0 & 38506.0 & 42980.0 & 47550.0 \\
\hline 1,3-Butadiene..... & $\mathrm{C}_{4} \mathrm{H}_{6}$ & 0 & 3625.6 & 3660.0 & 5836.0 & 8485.0 & 11508.0 & 14833.0 & 18408.0 & 22194.0 & 26160.0 & 30294.0 & 34560.0 & 38948.0 & 43442.0 & 48030.0 \\
\hline 1,2-Pentadiene & $\mathrm{C}_{5} \mathrm{H}_{8}$ & 0 & 4830 & 4890 & 7720 & 11150 & 15000 & 19320 & 23920 & 28800 & 34000 & 39380 & 44880 & 50700 & 56560 & 62550 \\
\hline cis-1,3-Pentadiene (cis-piperylene) ..... & $\mathrm{C}_{5} \mathrm{H}_{8}$ & 0 & 4323 & 4380 & 7000 & 10250 & 14040 & 18200 & 22800 & 27630 & 32700 & 38060 & 43560 & 49270 & 55160 & 61050 \\
\hline tran $\&-1,3-P e n t a d i e n e(t r a n s$-piperylene) & $\mathrm{C}_{5} \mathrm{H}_{8}$ & 0 & 4562 & 4620 & 7440 & 10850 & 14700 & 18970 & 23600 & 28530 & 33700 & 39050 & 44640 & 50310 & 56140 & 62100 \\
\hline 1,4-Pentadiene & $\mathrm{C}_{5} \mathrm{H}_{8}$ & 0 & 4920 & 4980 & 7840 & 11200 & 15120 & 19390 & 23920 & 28800 & 34000 & 39380 & 44880 & 50700 & 56560 & 62550 \\
\hline 2,3-Pentadiene & $\mathrm{C}_{5} \mathrm{H}_{8}$ & 0 & 4681 & 4740 & 7440 & 10700 & 14400 & 18550 & 23040 & 27900 & 33000 & 38280 & 43800 & 49530 & 55300 & 61350 \\
\hline 3-Methyl-1,2-butadiene & $\mathrm{C}_{5} \mathrm{H}_{8}$ & 0 & 4711 & 4740 & 7560 & 10900 & 14760 & 18970 & 23520 & 28350 & 33500 & 38830 & 44400 & 50180 & 56000 & 61950 \\
\hline 2-Methyl-1,3-butadiene, (isoprene) ..... & $\mathrm{C}_{5} \mathrm{H}_{8}$ & 0 & 4472 & 4530 & 7360 & 10850 & 14760 & 19110 & 23760 & 28710 & 33800 & 39270 & 44880 & 50570 & 56420 & 62400 \\
\hline
\end{tabular}


TABLE 5. Values of the heat capacity, for the ideal gaseous state, to $1,500^{\circ} \mathrm{K}$, for the $\mathrm{C}_{3}$ to $\mathrm{C}_{5}$ diolefins

\begin{tabular}{|c|c|c|c|c|c|c|c|c|c|c|c|c|c|c|c|c|}
\hline \multirow{3}{*}{ Compound (gas) } & \multirow{3}{*}{$\begin{array}{l}\text { For- } \\
\text { mula }\end{array}$} & \multicolumn{15}{|c|}{ Temperature in ${ }^{\circ} \mathrm{K}$} \\
\hline & & 0 & 298.16 & 300 & 400 & 500 & 600 & 700 & 800 & 900 & 1,000 & 1,100 & 1,200 & 1,300 & 1,400 & 1,500 \\
\hline & & \multicolumn{15}{|c|}{ Heat capacity, $C_{p}^{\circ}$, cal/deg mole } \\
\hline Propadiene (allene). & $\mathrm{C}_{3} \mathrm{H}_{4}$ & 0 & 14.10 & 14.16 & 17. 21 & 19.82 & 22.00 & 23.84 & 25.42 & 26.80 & 28.00 & 29.04 & 29.96 & 30.75 & 31.45 & 32.06 \\
\hline 1,2-Butadiene. ....... & $\mathrm{C}_{4} \mathrm{H}_{6}$ & 0 & 19.15 & 19.23 & 23.54 & 27.39 & 30.72 & 33.54 & 36.01 & 38.16 & 40.02 & 41.62 & 43.02 & 44.23 & 45.29 & 46. 20 \\
\hline 1,3-Butadiene . . & $\mathrm{C}_{4} \mathrm{H}_{6}$ & 0 & 19.01 & 19.11 & 24.29 & 28.52 & 31.84 & 34.55 & 36.84 & 38.81 & 40.52 & 42.02 & 43.32 & 44.47 & 45.47 & 46.34 \\
\hline 1,2-Pentadiene & $\mathrm{C}_{5} \mathrm{H}_{8}$ & 0 & 25.2 & 25.3 & 31.4 & 36.5 & 40.8 & 44.5 & 47.7 & 50.4 & 52.8 & 54.9 & 56.7 & 58.2 & 59.6 & 60.7 \\
\hline cis-1,3-Pentadiene (cis-piperylene) ..... & $\mathrm{C}_{5} \mathrm{H}_{8}$ & 0 & 22.6 & 22.7 & 29.5 & 35.3 & 39.9 & 43.8 & 47.0 & 49.8 & 52.2 & 54.3 & 56.1 & 57.7 & 59.1 & 60.3 \\
\hline trans-1,3-Pentadiene(trans-piperylene). & $\mathrm{C}_{5} \mathrm{H}_{8}$ & 0 & 24.7 & 24.9 & 31.2 & 38.6 & 40.9 & 44.6 & 47.7 & 50.3 & 52.6 & 54.7 & 56.4 & 58.0 & 59.3 & 60.5 \\
\hline 1,4-Pentadiene & $\mathrm{C}_{5} \mathrm{H}_{8}$ & 0 & 25.1 & 25.2 & 31.3 & 36.5 & 40.8 & 44.4 & 47.6 & 50.3 & 52.7 & 54.7 & 56.5 & 58.1 & 59.4 & 60.6 \\
\hline 2,3-Pentadiene & $\mathrm{C}_{5} \mathrm{H}_{8}$ & 0 & 24.2 & 24.3 & 29.9 & 35.0 & 39.4 & 43.2 & 46.6 & 49.5 & 52.0 & 54.2 & 56.1 & 57.7 & 59.1 & 60.3 \\
\hline 3-Methyl-1,2-butadiene.... & $\mathrm{C}_{5} \mathrm{H}_{8}$ & 0 & 25.2 & 25.3 & 31.0 & 36.0 & 40.3 & 44.0 & 47.2 & 50.0 & 52.4 & 54.5 & 56.3 & 57.9 & 59.3 & 60.5 \\
\hline 2-Methyl-1,3-butadiene (isoprene) ..... & $\mathrm{C}_{5} \mathrm{H}_{3}$ & 0 & 25.0 & 25.2 & 31.8 & 37.1 & $4 \mathrm{i} .4$ & 45.0 & 48.0 & 50.6 & 52.9 & 54.9 & 56.6 & 58.2 & 59.5 & 60.6 \\
\hline
\end{tabular}

\section{Styrene and the Methylstyrenes}

For styrene and the several methylstyrenes, the values of the heat-content function, free-energy function, and heat capacity were taken from reference [26]. As usual, the value of the entropy is given by the value of the heat-content function less the value of the free-energy function, and the heat content is obtained by multiplying the value of the heat-content function by the temperature.

The resulting values of the thermodynamic functions for styrene and the methylstyrenes are given in tables 6 to 10 , inclusive, which give values of the following properties, respectively, from $0^{\circ}$ to $1,500^{\circ} \mathrm{K}$ : Heat-content function, free-energy function, entropy, heat content, and heat capacity.

TABLE 6. Values of the heat-content function, for the ideal gaseous state, to $1,500^{\circ} \mathrm{K}$, for styrene, $\mathrm{C}_{8}$, and the methylstyrenes, $\mathrm{C}_{9}$

\begin{tabular}{|c|c|c|c|c|c|c|c|c|c|c|c|c|c|c|c|c|}
\hline \multirow{3}{*}{ (gas) } & \multirow{3}{*}{$\begin{array}{l}\text { Form- } \\
\text { ula }\end{array}$} & \multicolumn{15}{|c|}{ Temperature in ${ }^{\circ} \mathrm{K}$} \\
\hline & & 0 & 298.16 & 300 & 400 & 500 & 600 & 700 & 800 & 900 & 1,000 & 1,100 & 1,200 & 1,300 & 1,400 & 1,500 \\
\hline & & \multicolumn{15}{|c|}{ Heat-content function, $\left(H^{\circ}-H_{0}^{\circ}\right) / T$, in cal/deg mole } \\
\hline $\begin{array}{l}\text { Ethenylbenzene (styrene; vinylbenz- } \\
\text { ene: Phenylethylene) }\end{array}$ & $\mathrm{C}_{8} \mathrm{H}$ & 0 & 16.72 & 16. 79 & 21.07 & 25,32 & 29.28 & 32.92 & 36.22 & 39.22 & 41.95 & 44,44 & 46.70 & 48.77 & 50,67 & 5242 \\
\hline $\begin{array}{l}\text { Isopropenylbenzene } \quad(\alpha \text {-methylsty- } \\
\text { rene; 2-phenyl-1-propene) }\end{array}$ & $\mathrm{C}_{9} \mathrm{H}_{10}$ & 0 & 20.3 & 20.3 & 25.2 & 30.0 & 34.6 & 38.8 & 42.6 & 46.1 & 49.3 & 52.2 & 54.9 & & 59.6 & 61.6 \\
\hline $\begin{array}{l}\text { cis-1-Propenylbenzene (cis- } \beta \text {-methyl- } \\
\text { styrene; cis-1-phenyl-1-propene) }\end{array}$ & $\mathrm{C}_{2} \mathrm{H}_{10}$ & 0 & 20.3 & 20.3 & 25.2 & 30.0 & 34.6 & 38.8 & 42.6 & 46.1 & 49.3 & 52.2 & 54.9 & 57.3 & 59.6 & 61.6 \\
\hline $\begin{array}{l}\text { trans - } 1 \text { - propenylbenzene (trans - } \beta \text { - } \\
\text { methylstyrene; trans -1-phenyl-1- } \\
\text { propene) }\end{array}$ & $\mathrm{C}_{9} \mathrm{H}_{10}$ & 0 & 19. 9 & 20.0 & 25.1 & 30.0 & 34.6 & 38.9 & 42.7 & 46.2 & 49.5 & 52.4 & 55.1 & 57.5 & 59.8 & 61.8 \\
\hline $\begin{array}{l}\text { 1-Methyl-2-ethenylbenzene (o-methyl- } \\
\text { styrene) }\end{array}$ & $\mathrm{C}_{9} \mathrm{H}_{1}$ ก & 0 & 20.3 & 20.3 & 25. 2 & 30.0 & 34.6 & 38.8 & 42.6 & 46.1 & 49.3 & 52.2 & 54. 9 & 57.3 & 59.6 & 61.6 \\
\hline $\begin{array}{l}\text { 1-Methyl-3-ethenylbenzene ( } m \text {-meth- } \\
\text { ylstyrene) }\end{array}$ & $\mathrm{C}_{9} \mathrm{H}_{10}$ & 0 & 20.3 & 20.3 & 25. 2 & 30.0 & 34.6 & 38.8 & 42.6 & 46.1 & 49.3 & 52.2 & 54.9 & 57.3 & 59.6 & 61.6 \\
\hline $\begin{array}{l}\text { 1-Methyl-4-ethenylbenzene ( } p \text {-meth- } \\
\text { ylstyrene) }\end{array}$ & $\mathrm{C}_{\vartheta} \mathrm{H}_{10}$ & 0 & 20.3 & 20.3 & 25.2 & 30.0 & 34.6 & 38.8 & 42. 6 & 46.1 & 49.3 & 52.2 & 54.9 & 57.3 & 59.6 & 61.6 \\
\hline
\end{tabular}


TABLE 7. Values of the free-energy function, for the ideal gaseous state, to $1,500^{\circ} \mathrm{K}$, for styrene, $\mathrm{C}_{8}$, and the methylstyrenes, $\mathrm{C}_{9}$

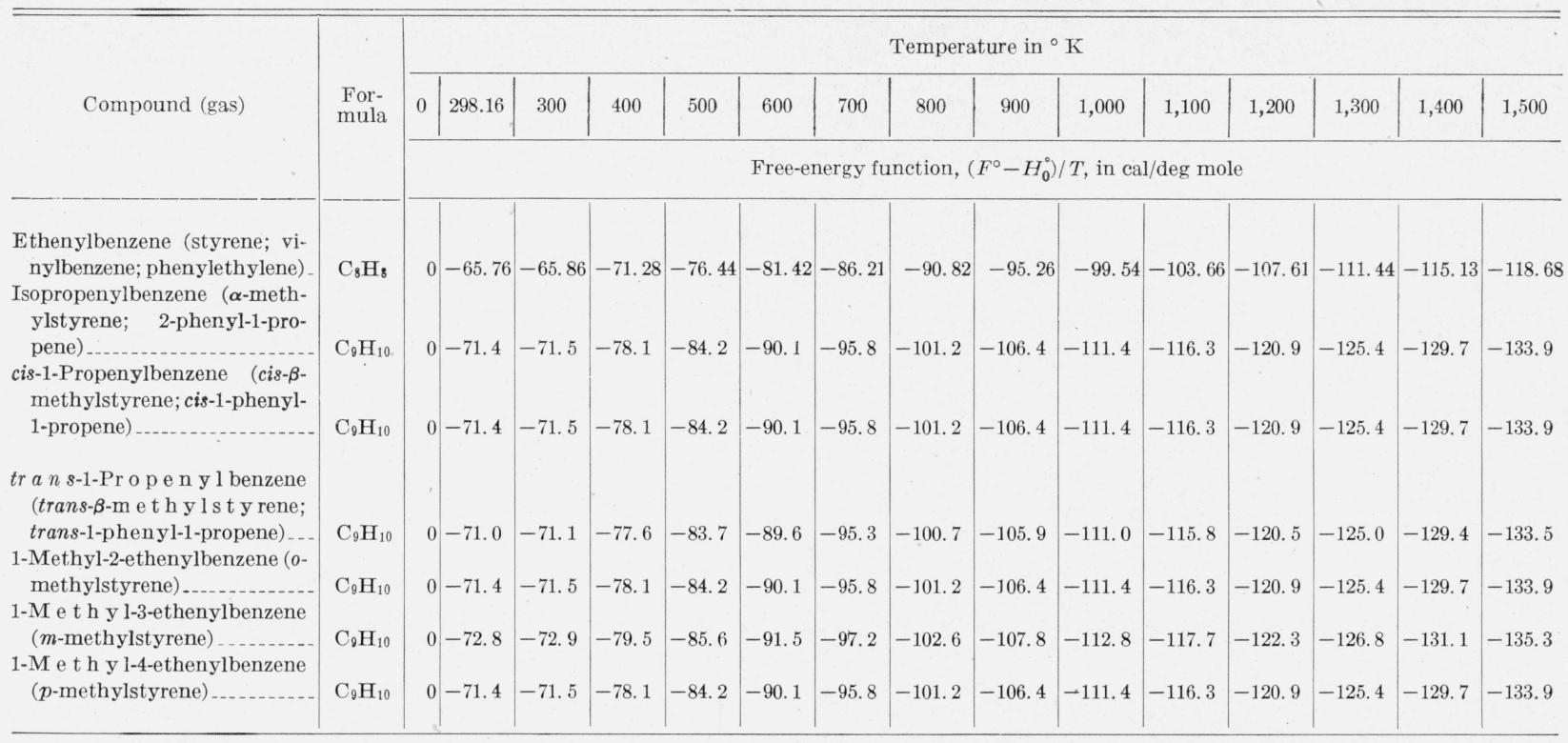

TABLE 8. Values of the entropy, for the ideal gaseous state, to $1,500^{\circ} \mathrm{K}$, for styrene, $\mathrm{C}_{8}$, and the methylstyrenes, $\mathrm{C}_{9}$

\begin{tabular}{|c|c|c|c|c|c|c|c|c|c|c|c|c|c|c|c|c|}
\hline \multirow{3}{*}{ Compound (gas) } & \multirow{3}{*}{$\begin{array}{l}\text { For- } \\
\text { mula }\end{array}$} & \multicolumn{15}{|c|}{ Temperature in ${ }^{\circ} \mathrm{K}$} \\
\hline & & 0 & 298.16 & 300 & 400 & 500 & 600 & 700 & 800 & 900 & 1,000 & 1,100 & 1,200 & 1,300 & 1,400 & 1,500 \\
\hline & & \multicolumn{15}{|c|}{ Entropy, $S^{\circ}$, in cal/deg mole } \\
\hline $\begin{array}{l}\text { Ethenylbenzene (styrene; vinylben- } \\
\text { zene; phenylethylene) } \\
\text { Isopropenylbenzene }(\alpha \text {-methylstyrene; }\end{array}$ & $\mathrm{C}_{8} \mathrm{H}_{8}$ & 0 & 82.48 & 82.65 & 92.35 & 101.76 & 110.70 & 119.13 & 127.04 & 134. 48 & 141. 49 & 148. 10 & 154.31 & 160. 21 & 165.80 & 171.10 \\
\hline $\begin{array}{l}\text { 2-phenyl-1-propene) } \\
\text { cis-1-Propenylbenzene (cis- } \beta \text {-methyl- }\end{array}$ & $\mathrm{C}_{9} \mathrm{H}_{10}$ & 0 & 91.7 & 91.8 & 103.3 & 114. 2 & 124. 7 & 134.6 & 143.8 & 152.5 & 160.7 & 168.5 & 175.8 & 182.7 & 189.3 & 195.5 \\
\hline styrene; cis-1-phenyl-1-propene) & $\mathrm{C}_{9} \mathrm{H}_{10}$ & 0 & 91. 7 & 91.8 & 103.3 & 114. 2 & 124.7 & 134.6 & 143.8 & 152.5 & 160.7 & 167.5 & 175.8 & 182.7 & 189.3 & 195.5 \\
\hline pene) & $\mathrm{C}_{2} \mathrm{H}_{10}$ & 0 & 90.9 & 91.1 & 102.7 & 113.7 & 124.2 & 134. 2 & 143.4 & 152.1 & 160.5 & 168.2 & 175.6 & 182.5 & 189. 2 & 195. 3 \\
\hline $\begin{array}{l}\text { 1-Methyl-2-ethenylbenzene (o-methyl- } \\
\text { styrene) }\end{array}$ & $\mathrm{C}_{9} \mathrm{H}_{10}$ & 0 & 91.7 & 91.8 & 103.3 & 114. 2 & 124.7 & 134.6 & 143.8 & 152.5 & 160.7 & 168.5 & 175.8 & 182. 7 & 189.3 & 195.5 \\
\hline $\begin{array}{l}\text { 1-Methyl-3-ethenylbenzene ( } m \text {-meth- } \\
\text { ylstyrene) }\end{array}$ & $\mathrm{C}_{9} \mathrm{H}_{10}$ & 0 & 93.1 & 93.2 & 104.7 & 115. 6 & 126.1 & 136.0 & 145.2 & 153.9 & 162.1 & 169.9 & 177.2 & 184.1 & 190.7 & 196.7 \\
\hline $\begin{array}{l}\text { 1-Methyl-4-ethenylbenzene ( } p \text {-methyl- } \\
\text { styrene) }\end{array}$ & $\mathrm{C}_{9} \mathrm{H}_{10}$ & 0 & 91. 7 & 91.8 & 103.3 & 114. 2 & 124.7 & 134.6 & 143.8 & 152.5 & 160.7 & 168.5 & 175.8 & 182.7 & 189.3 & 195.5 \\
\hline
\end{tabular}


TABLE 9. Values of the heat content, for the ideal gaseous state, to $1,500^{\circ} \mathrm{K}$, for styrene, $\mathrm{C}_{8}$, and the methyl-styrenes, $\mathrm{C}_{9}$

\begin{tabular}{|c|c|c|c|c|c|c|c|c|c|c|c|c|c|c|c|c|}
\hline \multirow{3}{*}{ Compound (gas) } & \multirow{3}{*}{$\begin{array}{l}\text { For- } \\
\text { mula }\end{array}$} & \multicolumn{15}{|c|}{ Temperature in ${ }^{\circ} \mathrm{K}$} \\
\hline & & 0 & 298.16 & 300 & 400 & 500 & 600 & 700 & 800 & 900 & 1,000 & 1,100 & 1,200 & 1,300 & 1,400 & 1,500 \\
\hline & & \multicolumn{15}{|c|}{ Heat content $\left(H^{\circ}-H_{0}^{\circ}\right)$, in cal $/$ mole } \\
\hline $\begin{array}{l}\text { Ethenylbenzene (styrene; vinylben- } \\
\text { zene; phenylethylene) }\end{array}$ & $\mathrm{C}_{8} \mathrm{H}_{8}$ & \multirow[t]{2}{*}{0} & 4,985 & \multirow{2}{*}{5,037} & 8,428 & 12,660 & 17,568 & 23,044 & 28,976 & \multirow{2}{*}{35,298} & \multirow{2}{*}{41,950} & \multirow{2}{*}{48,880} & \multirow{2}{*}{56,040} & \multirow{2}{*}{63,400} & \multirow{2}{*}{70,940} & 78,630 \\
\hline $\begin{array}{l}\text { Isopropenylbenzene ( } \alpha \text {-methylstyrene; } \\
\text { 2-phenyl-1-propene) }\end{array}$ & $\mathrm{C}_{9} \mathrm{H}_{10}$ & & 6,050 & & 10,080 & 15,000 & 20,760 & 27,160 & 34,080 & & & & & & & 92,400 \\
\hline $\begin{array}{l}\text { cis-1-Propenylbenzene (cis- } \boldsymbol{\beta} \text {-methyl- } \\
\text { styrene; cis-1-phenyl-1-propene) }\end{array}$ & $\mathrm{C}_{9} \mathrm{H}_{10}$ & 0 & 6,050 & 6,090 & 10,080 & 15,000 & 20,760 & 27,160 & 34,080 & 41,490 & 49,300 & 57,400 & 65,900 & 74,500 & 83,400 & 92,400 \\
\hline propene) & $\mathrm{C}_{9} \mathrm{H}_{10}$ & 0 & 5,930 & 6,000 & 10,040 & 15,000 & 20,760 & 27,230 & 34,160 & 41,580 & 49,500 & 57,600 & 66,100 & 74,800 & 83,700 & 92,700 \\
\hline $\begin{array}{l}\text { 1-Methyl-2-ethenylbenzene (o-methyl- } \\
\text { styrene) }\end{array}$ & $\mathrm{C}_{2} \mathrm{H}_{10}$ & 0 & 6,050 & 6,090 & 10,080 & 15,000 & 20,760 & 27,160 & 34,080 & 41,490 & 49,300 & 57,400 & 65,900 & 74,500 & 83,400 & 92,400 \\
\hline $\begin{array}{l}\text { 1-Methyl-3-ethenylbenzene ( } m \text {-methyl- } \\
\text { styrene) }\end{array}$ & $\mathrm{C}_{9} \mathrm{H}_{10}$ & 0 & 6,050 & 6,090 & 10,080 & 15,000 & 20,760 & 27,160 & 34,080 & 41,490 & , 49,300 & 57,400 & 65,900 & 74,500 & 83,400 & 92,400 \\
\hline $\begin{array}{l}\text { 1-Methyl-4-ethenylbenzene ( } p \text {-methyl- } \\
\text { styrene) }\end{array}$ & $\mathrm{C}_{9} \mathrm{H}_{10}$ & 0 & 6,050 & 6,090 & 10,080 & 15,000 & 20,760 & 27,160 & 34,080 & 41,490 & 49,300 & 57,400 & 65,900 & 74,500 & 83,400 & 92,400 \\
\hline
\end{tabular}

TABLE 10. Values of the heat capacity, for the ideal gaseous state, to $1,500^{\circ} \mathrm{K}$, for styrene, $\mathrm{C}_{8}$, and the methylstyrenes, $\mathrm{C}_{9}$

\begin{tabular}{|c|c|c|c|c|c|c|c|c|c|c|c|c|c|c|c|c|}
\hline \multirow{3}{*}{ Compound (gas) } & \multirow{3}{*}{$\begin{array}{l}\text { Form- } \\
\text { ula }\end{array}$} & \multicolumn{15}{|c|}{ Temperature in ${ }^{\circ} \mathrm{K}$} \\
\hline & & 0 & 298.16 & 300 & 400 & 500 & 600 & 700 & 800 & 900 & 1,000 & 1,100 & 1,200 & 1,300 & 1,400 & 1,500 \\
\hline & & \multicolumn{15}{|c|}{ Heat capacity, $C_{p}^{\circ}$, in cal/deg mole } \\
\hline \multicolumn{17}{|c|}{ 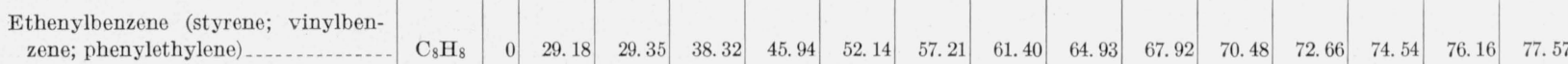 } \\
\hline $\begin{array}{l}\text { Isopropenylbenzene ( } \alpha \text {-methylstyrene; } \\
\text { 2-phenyl-1-propene) } \\
\text { cis-1-Propenylbenzene (cis- } \beta \text {-methyl- }\end{array}$ & $\mathrm{C}_{9} \mathrm{H}_{10}$ & 0 & 34.7 & 34.9 & 44.8 & 53.5 & 60.7 & 66.8 & 71.8 & 76.1 & 79.8 & 82.9 & 85.6 & 87. 9 & 89.9 & 91.6 \\
\hline styrene; cis-1-phenyl-1-propene) & $\mathrm{C}_{9} \mathrm{H}_{10}$ & 0 & 34.7 & 34.9 & 44.8 & 53.5 & 60.7 & 66.8 & 71.8 & 76.1 & 79.8 & 82.9 & 85.6 & 87.9 & 89.9 & 91.6 \\
\hline $\begin{array}{l}\text { trans-1-Propenylbenzene (trans- } \beta \text { - } \\
\text { methylstyrene; trans-1-phenyl-1- } \\
\text { propene) }\end{array}$ & $\mathrm{C}_{9} \mathrm{H}_{10}$ & 0 & 34.9 & 35.1 & 45. 2 & 54.0 & 61.2 & 67.2 & 72.2 & 76.4 & 80.0 & 83.1 & 85.8 & 88.1 & 90.0 & 91.7 \\
\hline $\begin{array}{l}\text { 1-Methyl-2-ethenylbenzene (o-methyl- } \\
\text { styrene) }\end{array}$ & $\mathrm{C}_{9} \mathrm{H}_{10}$ & 0 & 34.7 & 34.9 & 44.8 & 53.5 & 60.7 & 66.8 & 71.8 & 76.1 & 79.8 & 82.9 & 85.6 & 87. 9 & 89.9 & 91.6 \\
\hline $\begin{array}{l}\text { 1-Methyl-3-ethenylbenzene ( } m \text {-meth- } \\
\text { ylstyrene) }\end{array}$ & $\mathrm{C}_{9} \mathrm{H}_{10}$ & 0 & 34.7 & 34.9 & 44.8 & 53.5 & 60.7 & 66.8 & 71.8 & 76.1 & 79.8 & 82.9 & 85.6 & 87.9 & 89.9 & 91.6 \\
\hline $\begin{array}{l}\text { 1-Methyl-4-ethenylbenzene ( } p \text {-methyl- } \\
\text { styrene) }\end{array}$ & $\mathrm{C}_{9} \mathrm{H}_{10}$ & 0 & 34.7 & 34.9 & 44.8 & 53.5 & 60.7 & 66.8 & 71.8 & 76.1 & 79.8 & 82.9 & 85.6 & 87.9 & 89.9 & 91.6 \\
\hline
\end{tabular}




\section{Heat of Formation, Free Energy of Formation, and Equilibrium Constant of Formation}

For the standard heat of formation from the elements at $25^{\circ} \mathrm{C}$, values for the diolefins, $\mathrm{C}_{3}$ to $\mathrm{C}_{5}$, were obtained as follows: 1,3-Butadiene, from reference [27]; 1,2-butadiene, from reference [28]; propadiene, cis- and trans-1,3-pentadiene, and 1,4pentadiene, from combination of the data on heats of hydrogenation from references [36, 37] with the values for the heats of formation of the corresponding paraffin hydrocarbons from reference [29]; by calculation, using the relations between energy content and molecular structure given in references $[29,30,31,32,33]$. The values so calculated are believed to be uncertain by not more than about $\pm 0.75 \mathrm{kcal} / \mathrm{mole}$.

For the standard heat of formation from the elements at $25^{\circ} \mathrm{C}$, values for styrene and the methylstyrenes were obtained as follows:

Ethenylbenzene (styrene, vinylbenzene, or phenylethylene), from reference [28]; isopropenylbenzene ( $\alpha$-methylstyrene or 2-phenyl-1-propene), from reference [34]; cis-1-propenylbenzene (cis- $\beta$ methylstyrene or cis-1-phenyl-1-propene), trans-1propenylbenzene (trans- $\beta$-methylstyrene or trans1-phenyl-1-propene), 1-methyl-2-ethenylbenzene (o-methylstyrene), 1-methyl-3-ethenylbenzene, (m-methylstyrene), and 1-methyl-4-ethenylbenzene ( $p$-methylstyrene) by calculation, using the relations between energy content and molecular structure given in references [29, 30, 31, 32, 33]. The values so calculated are believed to be uncertain by not more than about $\pm 0.75 \mathrm{kcal} / \mathrm{mole}$.

The method of calculating values of the standard heat of formation, the standard free energy of formation, and the logarithm of the equilibrium constant of formation for the different temperatures in the range $0^{\circ}$ to $1,500^{\circ} \mathrm{K}$, is the same as that described in section IV, 1 , of reference [35].

The resulting values for the formation of the given hydrocarbon in the gaseous state, from the elements carbon (solid, graphite) and hydrogen (gaseous), each in its thermodynamic standard state, are presented in tables 11 to 16 , inclusive, which give values of the following properties, for the $\mathrm{C}_{3}$ to $\mathrm{C}_{5}$ diolefins, styrene, and the methylstyrenes, to $1,500^{\circ} \mathrm{K}$ : Heat of formation, free energy of formation, and logarithm of the equilibrium constant of formation. It should be noted that the uncertainties in the values for the pentadienes and the methylstyrenes are such as to make the values of the logarithm (to the base 10) of the equilibrium constants of formation for these compounds uncertain by as much as 0.3 to 0.5 , which is equivalent to a factor of 2 to 3 in the value of the equilibrium constant itself. 


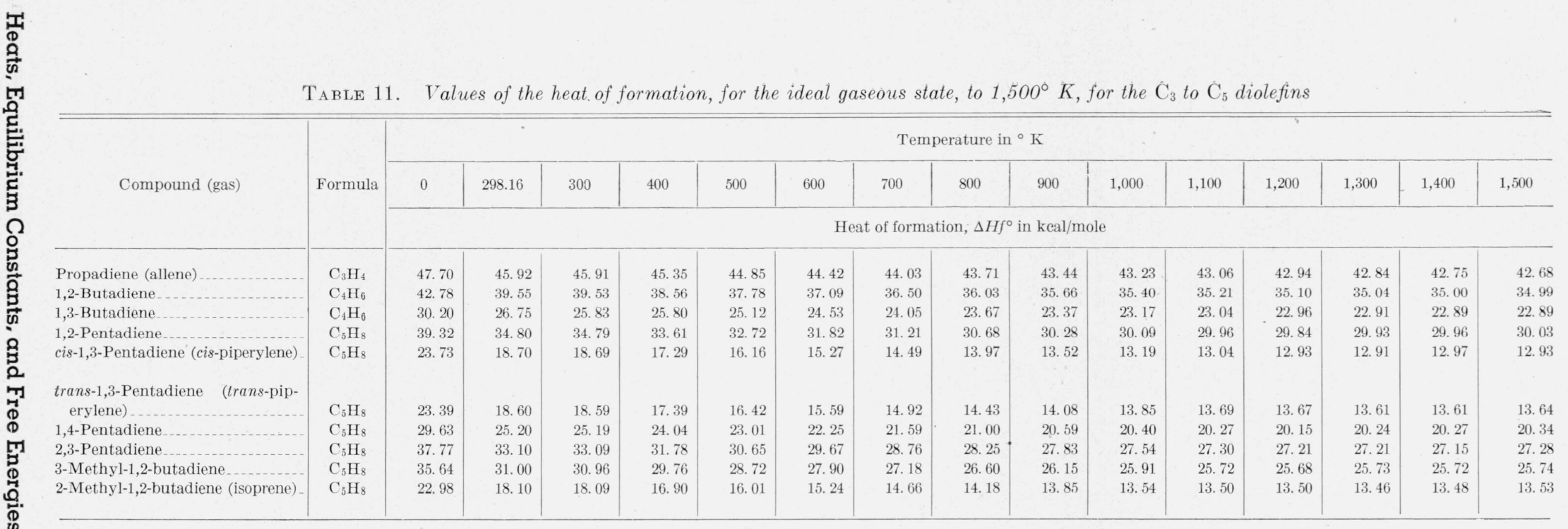

TABLE 12. Values of the free energy of formation, for the ideal gaseous state, to $1,500^{\circ} \mathrm{K}$, for the $\mathrm{C}_{3}$ to $\mathrm{C}_{5}$ diolefins

\begin{tabular}{|c|c|c|c|c|c|c|c|c|c|c|c|c|c|c|c|c|}
\hline \multirow{3}{*}{ Compound (gas) } & \multirow{3}{*}{ Formula } & \multicolumn{15}{|c|}{ Temperature in ${ }^{\circ} \mathrm{K}$} \\
\hline & & 0 & 298.16 & 300 & 400 & 500 & 600 & 700 & 800 & 900 & 1,000 & 1,100 & 1,200 & 1,300 & 1,400 & 1,500 \\
\hline & & \multicolumn{15}{|c|}{ Free energy of formation, $\Delta F f^{\circ}$ in kcal/mole } \\
\hline Propadiene (allene) & $\mathrm{C}_{3} \mathrm{H}_{4}$ & 47.70 & 48.37 & 48. 38 & 49. 29 & 50.33 & 51.48 & 52. 68 & 53. 93 & 55. 23 & 56.50 & 57. 86 & 39. 25 & 60.62 & 61.98 & 63.35 \\
\hline 1,2-Butadiene & $\mathrm{C}_{4} \mathrm{H}_{6}$ & 42.78 & 48. 21 & 48. 26 & 51.32 & 54.60 & 58.03 & 61.57 & 65.18 & 68.85 & 72.55 & 76. 26 & 80.01 & 83.75 & 87.50 & 91. 25 \\
\hline 1,3-Butadiene & $\mathrm{C}_{4} \mathrm{H}_{6}$ & 30.20 & 36.43 & 36.49 & 39.88 & 43.47 & 47. 20 & 51.02 & 54.90 & 58.82 & 62.78 & 66.74 & 70.72 & 74. 70 & 78.67 & 82.66 \\
\hline 1,2-Pentadiene & $\mathrm{C}_{5} \mathrm{H}_{8}$ & 39.32 & 50.29 & 50.38 & 55.77 & 61.44 & 67.28 & 73. 21 & 79. 27 & 85.41 & 91.53 & 97.70 & 103.85 & 109. 96 & 116.16 & 122. 22 \\
\hline cis-1,3-Pentadiene (cis-piperylene). & $\mathrm{C}_{5} \mathrm{H}_{8}$ & 23. 73 & 34.88 & 34.97 & 40.61 & 46.60 & 52.77 & 59. 09 & 65.44 & 71.98 & 78.44 & 84. 97 & 91.50 & 98.14 & 104. 77 & 111.13 \\
\hline trans-1,3-Pentadiene (trans-pip- & & & & & & & & & & & & & & & & \\
\hline erylene) & $\mathrm{C}_{5} \mathrm{H}_{8}$ & 23.39 & 35.07 & 35.17 & 40.87 & 46.91 & 53.09 & 59.38 & 65.74 & 72.18 & 78.70 & 85.18 & 91.64 & 98.19 & 104. 71 & 111.09 \\
\hline 1,4-Pentadiene & $\mathrm{C}_{5} \mathrm{H}_{8}$ & 29.63 & 40.69 & 40.78 & 46. 16 & 51.85 & 57.71 & 63.66 & 69.66 & 75.81 & 81.94 & 88.12 & 94. 28 & 100.53 & 106. 61 & 112.83 \\
\hline 2,3-Pentadiene & $\mathrm{C}_{5} \mathrm{H}_{8}$ & 37.77 & 49. 22 & 49.31 & 54.94 & 60.84 & 66. 99 & 73.27 & 79.64 & 86.11 & 92.58 & 99.12 & 105. 66 & 112.18 & 118.67 & 125.32 \\
\hline 3-Methyl-1,2-butadiene & $\mathrm{C}_{5} \mathrm{H}_{8}$ & 35.64 & 47.47 & 47.57 & 53. 29 & 59.31 & 65.46 & 71.77 & 78.23 & 84.70 & 91.25 & 97.76 & 104. 25 & 110.83 & 117.38 & 123.94 \\
\hline 2-Methyl-1,3-butadiene (isoprene) & $\mathrm{C}_{5} \mathrm{H}_{8}$ & 22.98 & 34.87 & 34.97 & 40.78 & 46.85 & 53.10 & 59.46 & 65.89 & 72.40 & 78.89 & 85.43 & 91.95 & 98.56 & 105.00 & 111.58 \\
\hline
\end{tabular}


TABLE 13. Values of the logarithm of the equilibrium constant of formation, for the ideal gaseous state, to $1,500^{\circ} \mathrm{K}$, for the $\mathrm{C}_{3}$ to $\mathrm{C}_{5}$ diolefins

\begin{tabular}{|c|c|c|c|c|c|c|c|c|c|c|c|c|c|c|c|c|}
\hline \multirow{3}{*}{ Compound (gas) } & \multirow{3}{*}{ Formula } & \multicolumn{15}{|c|}{ Temperature in ${ }^{\circ} \mathrm{K}$} \\
\hline & & 0 & 298.16 & 300 & 400 & 500 & 600 & 700 & 800 & 900 & 1,000 & 1,100 & 1,200 & 1,300 & 1,400 & 1,500 \\
\hline & & \multicolumn{15}{|c|}{ Logarithm of equilibrium constant of formation, $\log _{10} K f$} \\
\hline Propadiene (allene) & $\mathrm{C}_{3} \mathrm{H}_{4}$ & Infinite-. & -35.4519 & -35.2462 & -26.9316 & -22.0008 & -18.7503 & -16.4479 & -14.7338 & -13.4123 & -12.3488 & -11.5024 & -10.7904 & -10.1907 & -9.6747 & -9.2306 \\
\hline 1,2-Butadiene.. & $\mathrm{C}_{4} \mathrm{H}_{6}$ & ...do_... & -35.3377 & -35.1597 & -28.0379 & -23.8644 & -21.1356 & -19.2218 & -17.8050 & -16.7181 & -15.8564 & -15.1512 & -14.5710 & -14.0790 & -13.6600 & -13.2943 \\
\hline 1,3-Butadiene & $\mathrm{C}_{4} \mathrm{H}_{6}$ & .... do _... & -26.7004 & -26.5796 & -21.7868 & -19.0023 & -17.1941 & -15.9302 & -14.9974 & -14.2834 & -13.7205 & -13.2602 & -12.8799 & -12.5573 & -12.2809 & -12.8070 \\
\hline 1,2-Pentadiene... & $\mathrm{C}_{5} \mathrm{H}_{8}$ & ... do _. & -36.861 & -36.705 & -30.469 & -26.854 & -24.507 & -22.857 & -21.655 & -20.741 & -20.004 & -19.412 & -18.913 & -18.486 & -18.134 & -17.044 \\
\hline cis-1,3-Pentadiene(cis-piperylene) & $\mathrm{C}_{5} \mathrm{H}_{8}$ & ... do & -25.563 & -25.477 & -22.100 & -20.367 & -19.221 & -18.448 & -17.877 & -17.479 & -17.142 & -16.882 & -16.664 & -16.499 & -16.355 & -16.191 \\
\hline $\begin{array}{l}\text { trans-1,3-P enta diene } \quad \text { (trar } \\
\text { piperylene) }\end{array}$ & $\mathrm{C}_{5} \mathrm{H}_{8}$ & ...do do . & -25.707 & -25.624 & -22.332 & -20.502 & -19 & -18.538 & -17 & -17 & -17 . & -16.924 & -16.689 & -16 & -16.346 & -16.185 \\
\hline 1,4-Pentadiene & $\mathrm{C}_{5} \mathrm{H}_{8}$ & do & -29.824 & -29.712 & -25.219 & -22.663 & -21.021 & -19.875 & -19.030 & -18.410 & -17.908 & -17.508 & -17.171 & -16.901 & -16.643 & -16.439 \\
\hline 2,3-Pentadiene-........ & $\mathrm{C}_{5} \mathrm{H}_{8}$ & ... do & -36.074 & -35.925 & -30.017 & -26.592 & -24.401 & -22.875 & -21.756 & -20.910 & -20.233 & -19.693 & -19.243 & -18.859 & -18.526 & -18.259 \\
\hline 3-Methyl-1,2-butadi & $\mathrm{C}_{5} \mathrm{H}_{8}$ & ... do _. & -34.797 & -34.657 & -29.114 & -25.923 & -23.843 & -22.407 & -21.371 & -20.568 & -19.942 & -19.423 & -18.986 & -18.632 & -18.324 & -18.058 \\
\hline 2-Methyl-1,3-butadiene(isoprene) & $\mathrm{C}_{5} \mathrm{H}_{8}$ & ... do & -25.560 & -25.477 & -22.284 & -20.476 & -19.341 & -18.563 & -18.000 & -17.581 & -17.241 & -16.973 & -16.746 & -16.569 & -16.392 & -16.257 \\
\hline
\end{tabular}

TABLE 14. Values of the heat of formation, for the ideal gaseous state, to $1,500^{\circ} \mathrm{K}$, for styrene, $\mathrm{C}_{8}$, and the methylstyrenes, $\mathrm{C}_{9}$.

\begin{tabular}{|c|c|c|c|c|c|c|c|c|c|c|c|c|c|c|c|c|}
\hline \multirow{3}{*}{ Compound (gas) } & \multirow{3}{*}{ Formula } & \multicolumn{15}{|c|}{ Temperature in ${ }^{\circ} \mathrm{K}$} \\
\hline & & 0 & 298.16 & 300 & 400 & 500 & 600 & 700 & 800 & 900 & 1,000 & 1,100 & 1,200 & 1,300 & 1,400 & 1,500 \\
\hline & & \multicolumn{15}{|c|}{ Heat of formation, $\Delta H f^{\circ}$ in kcal/mole } \\
\hline \multirow{3}{*}{$\begin{array}{l}\text { Ethenylbenzene (styrene; vinyl- } \\
\text { benzene; phenylethylene) } \\
\text { Issopropenylbenzene ( } \alpha \text {-methyl- } \\
\text { styrene; 2-phenyl-1-propene) } \\
\text { cis-1-Propenylbenzene (cis- } \beta \text {-Me- } \\
\text { thylstyrene; cis-1-phenyl-1-pro- } \\
\text { pene) }\end{array}$} & \multirow{3}{*}{$\begin{array}{l}\mathrm{C}_{8} \mathrm{H}_{8} \\
\mathrm{C}_{8} \mathrm{H}_{10}\end{array}$} & 40.34 & 35.22 & 35.19 & 33.83 & 32.72 & 31.81 & 31.08 & 30.51 & 30. 09 & 29.83 & 29.69 & 29.63 & 29.61 & 29.63 & 29.68 \\
\hline & & 33.33 & 27.00 & 26.94 & 25.23 & 23.80 & 22.66 & 21.73 & 20. 99 & 20.45 & 20.13 & 19. 93 & 19.90 & 19.83 & 19. 95 & 19.93 \\
\hline & & 35.33 & 29.00 & 28.94 & 27.23 & 25.80 & 24.66 & 23.73 & 22.99 & 22.45 & 22.13 & 21.93 & 21.90 & 21.83 & & 21. 93 \\
\hline $\begin{array}{l}\text { trans-1-Propenylbenzene (trans- } \beta \text { - } \\
\text { methylstyrene; trans-1-phenyl- } \\
\text { 1-propene) }\end{array}$ & $\mathrm{C}_{9} \mathrm{H}_{10}$ & 34.45 & 28.00 & 27.97 & 26.31 & 24. 92 & 23.78 & 22. 92 & 22.19 & 21.66 & 21.45 & 21.27 & 21.26 & 21. 21 & 21.95 & 21.35 \\
\hline $\begin{array}{l}\text { 1- Methyl - } 2 \text { - ethenylbenzene) (o- } \\
\text { methylstyrene }\end{array}$ & $\mathrm{C}_{\vartheta} \mathrm{H}_{10}$ & 34.63 & 28.30 & 28.24 & 26. 53 & 25.10 & 23. 96 & 23.03 & 22. 29 & 21.75 & 21.43 & 21.23 & 21. 20 & 21.13 & 21.25 & 21. 23 \\
\hline $\begin{array}{l}\text { 1- Methyl - } 3 \text { - ethenylbenzene }(m \text { - } \\
\text { methylstyrene) }\end{array}$ & $\mathrm{C}_{\vartheta} \mathrm{H}_{10}$ & 33. 93 & 27.60 & 27.54 & 25.83 & 24.40 & 23. 26 & 22.33 & 21. 59 & 21.05 & 20.73 & 20.53 & 20. 50 & 20.43 & 20.55 & 20. 53 \\
\hline $\begin{array}{l}1 \text { - Methyl - } 4 \text { - ethenylbenzene ( } p \text {. } \\
\text { methylstyrene) }\end{array}$ & $\mathrm{C}_{9} \mathrm{H}_{10}$ & 33.73 & 27.40 & 27.34 & 25.63 & 24. 20 & 23. 06 & 22.13 & 21. 39 & 20.85 & 20. 53 & 20.33 & 20.30 & 20.23 & 20.35 & 20.33 \\
\hline
\end{tabular}




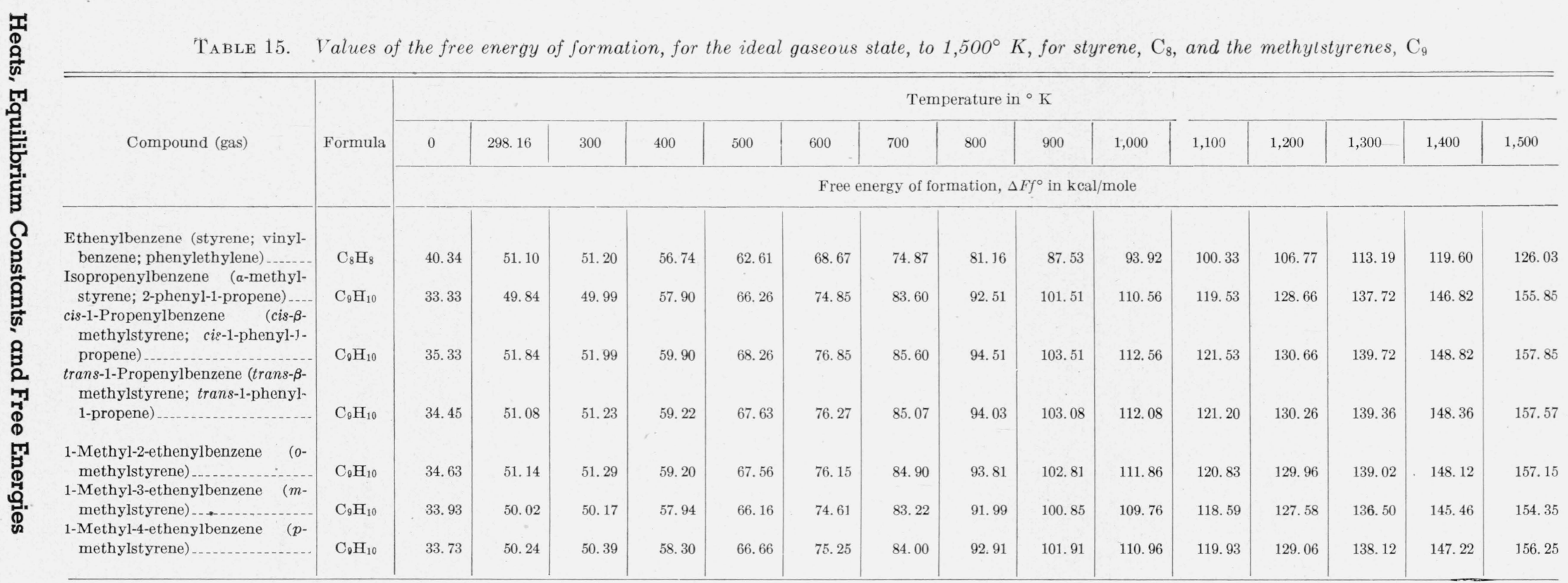

$\mathrm{T}_{\mathrm{ABLE}}$ 16. Values of the logarithm of the equilibrium constant of formation, for the ideal gaseous state, to $1,500^{\circ} \mathrm{K}$, for styrene, $\mathrm{C}_{8}$, and the methylstyrenes, $\mathrm{C}_{9}$

\begin{tabular}{|c|c|c|c|c|c|c|c|c|c|c|c|c|c|c|c|c|}
\hline \multirow{3}{*}{ Compound (gas) } & \multirow{3}{*}{ Formula } & \multicolumn{15}{|c|}{ Temperature in ${ }^{\circ} \mathrm{K}$} \\
\hline & & 0 & 298.16 & 300 & 400 & 500 & 600 & 700 & 800 & 900 & 1,000 & 1,100 & 1,200 & 1,300 & 1,400 & 1,500 \\
\hline & & \multicolumn{15}{|c|}{ Logarithm of equilibrium constant of formation, $\log _{10} \mathrm{Kf}$} \\
\hline $\begin{array}{l}\text { Ethenylbenzene (styrene; vinyl- } \\
\text { benzene; phenylethylene). }\end{array}$ & $\mathrm{C}_{8} \mathrm{H}_{8} \ldots$ & Infinite. & -37.4532 & -37.2963 & -31.0030 & -27.3649 & -25.0117 & -23.3762 & -22.1729 & -21.2543 & -20.5264 & -19.9330 & -19.4456 & -19.0295 & -18.6705 & -18.3630 \\
\hline $\begin{array}{l}\text { Isopropenylbenzene ( } \alpha \text {-methyl- } \\
\text { styrene; 2-phenyl-1-propene). }\end{array}$ & $\mathrm{C}_{9} \mathrm{H}_{10}$ & .... do _ & -36.531 & -36.416 & -31.636 & -28.963 & -27.266 & -26.101 & -25.272 & -24.649 & -24.162 & -23.749 & -23.433 & -23.153 & -22.919 & -22.707 \\
\hline $\begin{array}{l}\text { cis-1-Propenylbenzene (cis- } \beta \text { - } \\
\text { methylstyrene; cis-1-phenyl-1- } \\
\text { propene. }\end{array}$ & $\mathrm{C}_{9} \mathrm{H}_{10} \ldots$ & ... do & -37.998 & -37.874 & -32.729 & -29.837 & -27.994 & -26.726 & -25.818 & -25.134 & -24.600 & -24.146 & -23.797 & -23.490 & -23.231 & -22.998 \\
\hline $\begin{array}{l}\text { trans-1-Propenylbenzene (trans- } \\
\beta \text {-m ethylstyrene; trans- } \\
\text { 1-phenyl-1-propene). }\end{array}$ & $\mathrm{C}_{\vartheta} \mathrm{H}_{10} \ldots$ & .... do & -37.440 & -37.320 & -32.357 & -29.562 & -27.783 & -26.560 & -25.687 & -25.030 & -24.495 & -24.081 & -23.724 & -23.429 & -23.159 & -22.958 \\
\hline $\begin{array}{l}\text { 1-Methyl-2-ethen ylbenzene (o- } \\
\text { methylstyrene). }\end{array}$ & $\mathrm{C}_{9} \mathrm{H}_{10-.}$ & ... do & -37.484 & -37.364 & -32.346 & -29.531 & -27.739 & -26.560 & -25.627 & -24.965 & -24.446 & -24.007 & -23.670 & -23.352 & -23.122 & -22.896 \\
\hline $\begin{array}{l}\text { 1-Methyl-3-ethenylbenzene ( } m \text { - } \\
\text { methylstyrene). }\end{array}$ & $\mathrm{C}_{9} \mathrm{H}_{10}$ & -. do & -36.665 & -36.548 & -31.658 & -28.919 & -27.178 & -25.982 & -25.130 & -24.488 & -23.988 & -23.562 & -23.236 & -22.948 & -22.706 & -22.488 \\
\hline $\begin{array}{l}\text { 1-Methyl-4-ethenylbenzene ( } p \text { - } \\
\text { methylstyrene). }\end{array}$ & $\mathrm{C}_{9} \mathrm{H}_{10-}$ & .. do & -36.825 & -36.708 & -31.855 & -29.138 & -27.411 & -26.226 & -25.381 & -24.746 & -24.250 & -23.828 & -23.506 & -23.220 & -22.981 & -22.765 \\
\hline
\end{tabular}




\section{Free Energies and Equilibria of Some Reactions of Isomerization and Dehydrogenation}

In figure 1 are plotted, as a function of temperature, for the two butadienes, the amounts, in mole

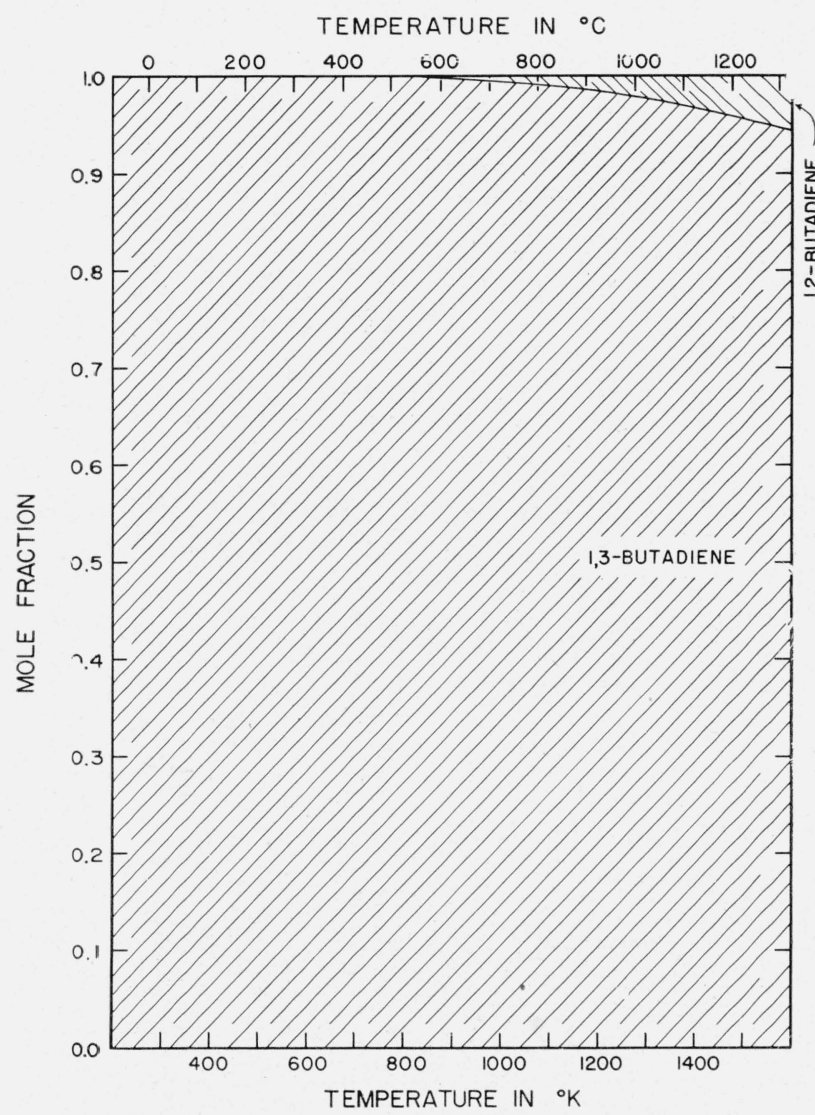

Figure 1. Equilibrium concentrations of the two butadienes.

The scale of ordinates measures the amount in mole fraction, and the scale of abscissas gives the temperature in degrees Kelvin and degrees Centigrade. The vertical width of a band at a given temperature measures the mole fraction of the given isomer present at equilibrium with all of its other isomers in the gaseous state.

fraction, of each of the isomers present at equilibrium with the other isomer in the gaseous state. The corresponding numerical values are given in table 17 .

In figure 2 are plotted, as a function of temperature, for the seven pentadienes, the amounts, in mole fraction, of each of the isomers present at equilibrium with the other isomers in the gaseous state. The corresponding numerical values are given in table 18.

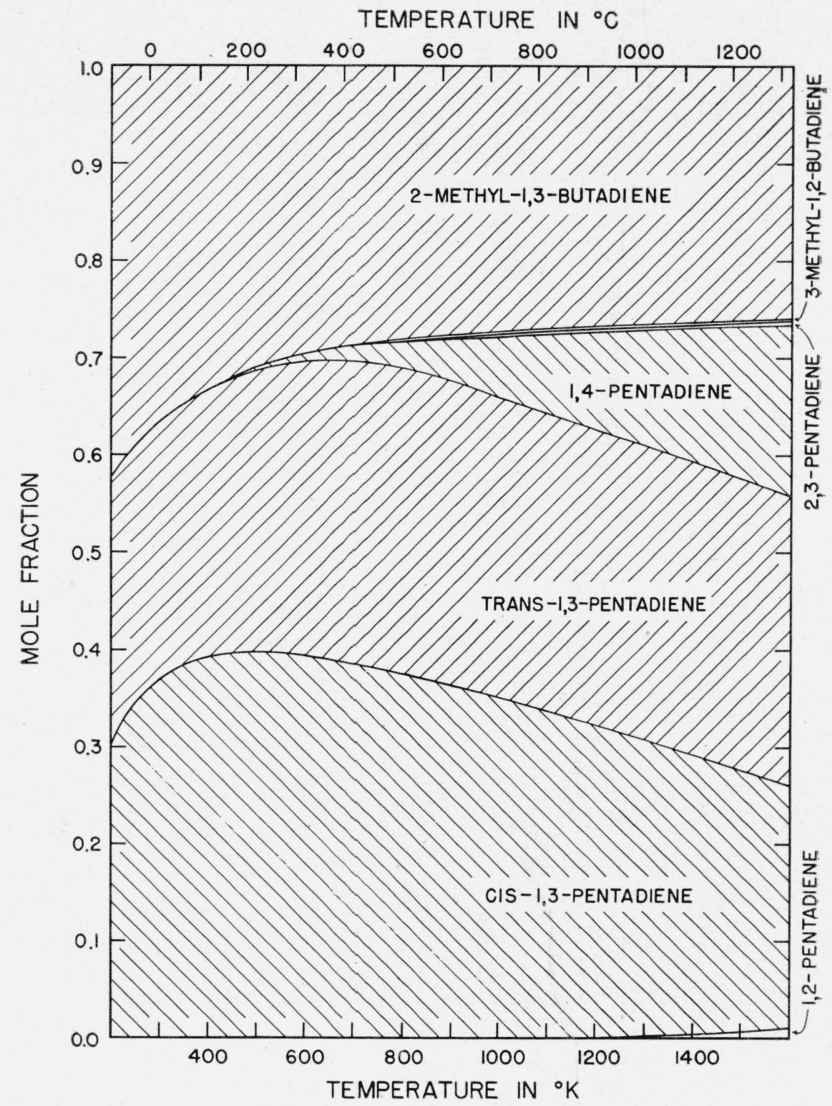

FIGURE 2. Equilibrium concentrations of the seven pentadienes.

The scale of ordinates measures the amount in mole fraction, and the scale of abscissas gives the temperature in degrees Kelvin and degrees Centigrade. The vertical width of a band at a given temperature measures the mole fraction of the given isomer present at equilibrium with all of its other isomers in the gaseous state.

TABLE 17. Equilibrium concentrations of the two butadienes in the ideal gaseous state to $1,500^{\circ} \mathrm{K}$

\begin{tabular}{|c|c|c|}
\hline \multirow{2}{*}{ Temperature } & \multicolumn{2}{|c|}{$\begin{array}{l}\text { Composition, in mole fraction } \\
\text { of the mixture of isomers at } \\
\text { at equlibrium }\end{array}$} \\
\hline & 1,2-Butadiene & 1,3-Butadiene \\
\hline$\therefore$ & & , \\
\hline $298.16 \ldots$ & 0.000 & 1.000 \\
\hline $300 \ldots \ldots$ & .000 & 1. 000 \\
\hline $400 \ldots \ldots$ & .000 & 1. 000 \\
\hline $500 \ldots \ldots$ & .000 & 1. 000 \\
\hline $600 \ldots \ldots$ & .000 & 1.000 \\
\hline $700 \ldots$ & .001 & .999 \\
\hline $800 \ldots$ & .002 & .998 \\
\hline $900 \ldots \ldots$ & .004 & .996 \\
\hline $1,000 \ldots \ldots$ & .007 & .993 \\
\hline $1,100 \ldots$. & .013 & .987 \\
\hline $1,200 \ldots$ & .020 & .980 \\
\hline $1,300 \ldots \ldots$ & .029 & .971 \\
\hline 1,400 & .040 & .960 \\
\hline $1,500 \ldots \ldots \ldots$ & .053 & .947 \\
\hline
\end{tabular}


TABLE 18. Equilibrium concentrations of the seven pentadienes in the ideal gaseous state to $1,500^{\circ} \mathrm{K}$

\begin{tabular}{|c|c|c|c|c|c|c|c|}
\hline \multirow{2}{*}{ Temperature } & \multicolumn{7}{|c|}{ Composition, in mole fraction, of the mixture of isomers at equilibrium } \\
\hline & 1,2-Pentadiene & $\begin{array}{c}\text { cis-1,3- } \\
\text { Pentadiene }\end{array}$ & $\begin{array}{c}\text { trans-1,3- } \\
\text { Pentadiene }\end{array}$ & 1,4-Pentadiene & 2,3-Pentadiene & $\begin{array}{l}\text { 3-Methyl-1,2- } \\
\text { butadiene }\end{array}$ & $\begin{array}{l}\text { 2-Methyl-1,3- } \\
\text { butadiene }\end{array}$ \\
\hline${ }^{\circ} K$ & & & & & & & \\
\hline $298.16 \ldots$ & 0.000 & 0.367 & 0.263 & 0.000 & 0.000 & 0.000 & 0.370 \\
\hline $300 \ldots$ & .000 & .369 & .262 & .000 & .000 & .000 & .369 \\
\hline $400 \ldots$ & .000 & .396 & .285 & .000 & .000 & .000 & .319 \\
\hline 500 & .000 & .398 & .291 & .002 & .000 & .000 & .309 \\
\hline $600 \ldots$ & .000 & .394 & .301 & .006 & .000 & .000 & .299 \\
\hline $700 \ldots$ & .000 & .382 & .311 & .014 & .000 & .000 & .293 \\
\hline $800 \ldots \ldots$ & .000 & .377 & .312 & .027 & .000 & .000 & .284 \\
\hline $900 \ldots$ & .000 & .357 & .319 & .042 & .000 & .000 & .282 \\
\hline $1000 \ldots \ldots$ & .000 & .351 & .308 & .060 & .000 & .001 & .280 \\
\hline $1100 \ldots \ldots$ & .001 & .338 & .306 & .080 & .001 & .001 & .274 \\
\hline $1200 \ldots$ & .002 & .323 & .305 & .100 & .001 & .002 & .267 \\
\hline 1300 & .003 & .308 & .302 & .122 & .001 & .002 & .262 \\
\hline 1400 & .005 & .286 & .293 & .148 & .002 & .003 & .263 \\
\hline 1500 & .007 & .287 & .291 & .162 & .0035 & .004 & .246 \\
\hline
\end{tabular}

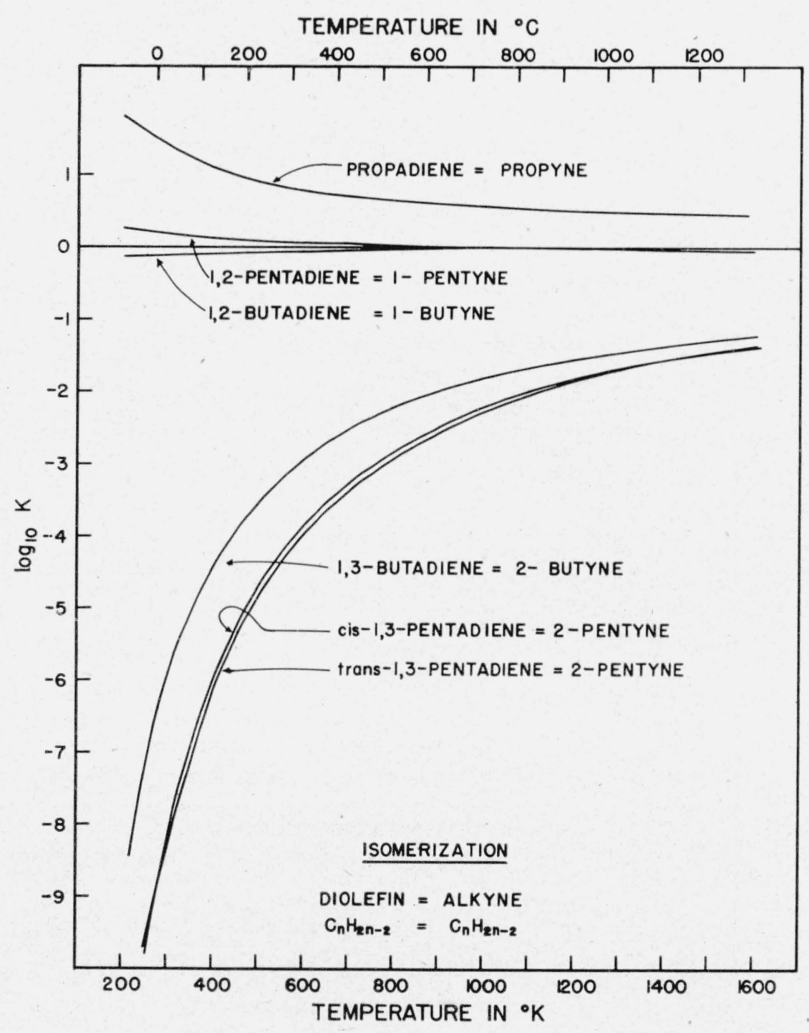

Figure 3. Dehydrogenation of monoolefins to diolefins.

The scale of ordinates gives the value of the logarithm (to the base 10) o the equilibrium constant for the reaction of dehydrogenating a given monoole ${ }^{*}$ fin to a given diolefin, in the gaseous state. The scale of abscissas gives the temperature in degrees Kelvin. The several curves refer to the following reactions in the gasesous state: $\mathrm{A}$, Propylene=propadiene+hydrogen; $\mathrm{B}$, 1-butene $=1,3$-butadiene + hydrogen; C, cis-2-butene $=1,3$-butadiene + hydrogen; D, trans-2-butene=1,3-butadiene+hydrogen; E, isobutene=1,3-butadiene+hydrogen.

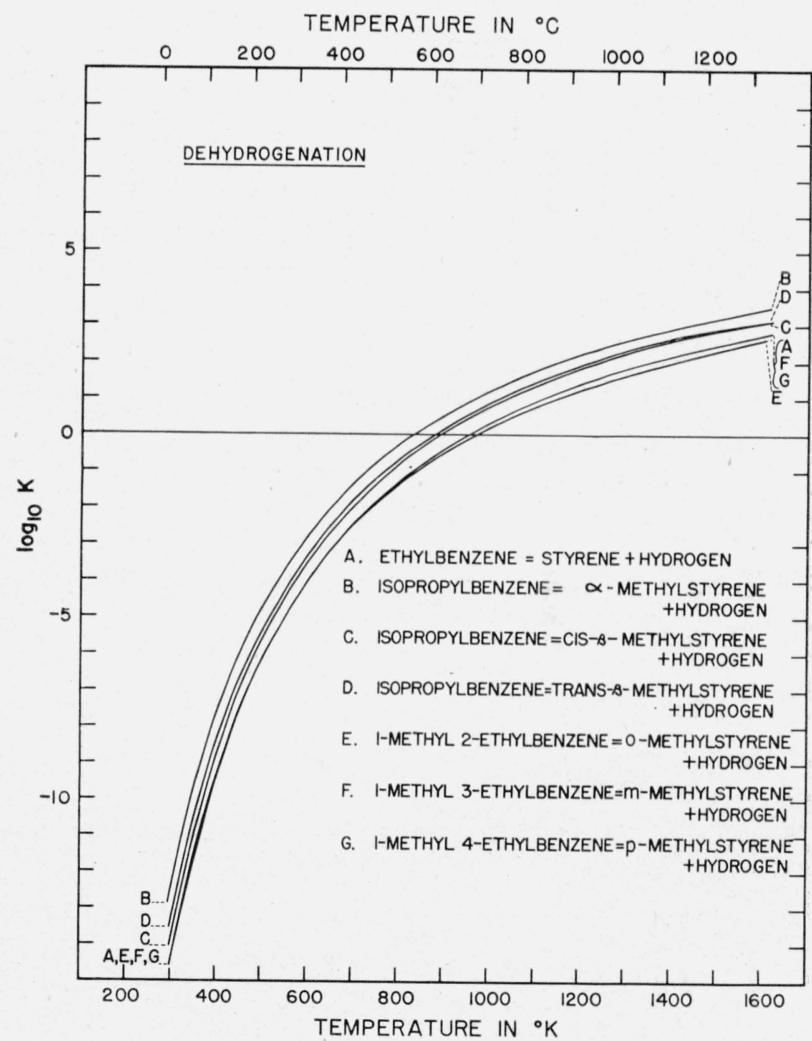

Figure 4. Isomerization of diolefins to acetylenes.

The scale of ordinates gives the value of the logarithm (to the base 10) of the equilibrium constant for the reaction of isomerization of a given diolefin to a given acetylene hydrocarbon, in the gaseous state. The scale of abscissas gives the temperature in degrees Kelvin. The several curves are for the following reactions in the gaseous state, as indicated: Propadiene=propyne; $1,2-$ butadiene=1-butyne; 1,2-pentadiene=1-pentyne; 1,3-butadiene=2-butyne; cis-1,3-pentadiene $=2$-pentyne; trans-1,3-pentadiene $=2$-pentyne. 
TABLE 19. Equilibrium concentrations of the six methylstyrenes in the ideal gaseous state to $1,500^{\circ} \mathrm{K}$

\begin{tabular}{|c|c|c|c|c|c|c|}
\hline \multirow{2}{*}{ Temperature } & \multicolumn{6}{|c|}{ Composition, in mole fraction, of the mixture of isomers, at equilibrium } \\
\hline & $\begin{array}{l}\alpha \text {-Methylsty- } \\
\text { rene }\end{array}$ & $\begin{array}{l}\text { cis- } \boldsymbol{\beta} \text {-Methyl- } \\
\text { styrene }\end{array}$ & $\begin{array}{l}\text { trans- } \boldsymbol{\beta}-\mathrm{Me}- \\
\text { thylstyrene }\end{array}$ & $\begin{array}{l}\text { o-Methylsty- } \\
\text { rene }\end{array}$ & $\begin{array}{l}m \text {-Methylsty- } \\
\text { rene }\end{array}$ & $\begin{array}{l}p \text {-Methylsty- } \\
\text { rene }\end{array}$ \\
\hline${ }^{\circ} \mathrm{K}$ & & & & & & \\
\hline $298.16 \ldots \ldots \ldots$ & 0.414 & 0.014 & 0.051 & 0.046 & 0.265 & 0.210 \\
\hline $300 \ldots \ldots$ & .396 & .014 & .049 & .045 & .293 & .203 \\
\hline $400 \ldots$ & .329 & .027 & .062 & .064 & .319 & .199 \\
\hline $500 \ldots$ & .292 & .039 & .073 & .079 & .322 & .195 \\
\hline $600 \ldots$ & .265 & .050 & .081 & .089 & .325 & .190 \\
\hline $700 \ldots$ & .247 & .059 & .086 & .097 & .325 & .186 \\
\hline $800 \ldots$ & .234 & .066 & 090 & .103 & .325 & .182 \\
\hline $900 \ldots . .$. & .224 & .073 & .093 & .108 & .323 & .180 \\
\hline $1,000 \ldots \ldots$ & .215 & .079 & .096 & .112 & .322 & .176 \\
\hline $1,100 \ldots$. & .208 & .083 & .100 & .115 & .320 & .174 \\
\hline $1,200 \ldots$ & .202 & .088 & .103 & .117 & .319 & .171 \\
\hline $1,300 \ldots \ldots$ & .198 & .091 & .105 & .120 & .318 & .168 \\
\hline $1,400 \ldots \ldots$ & .192 & .093 & .110 & .120 & .319 & .166 \\
\hline $1,500 \ldots$ & .191 & .097 & .107 & .123 & .315 & .167 \\
\hline
\end{tabular}

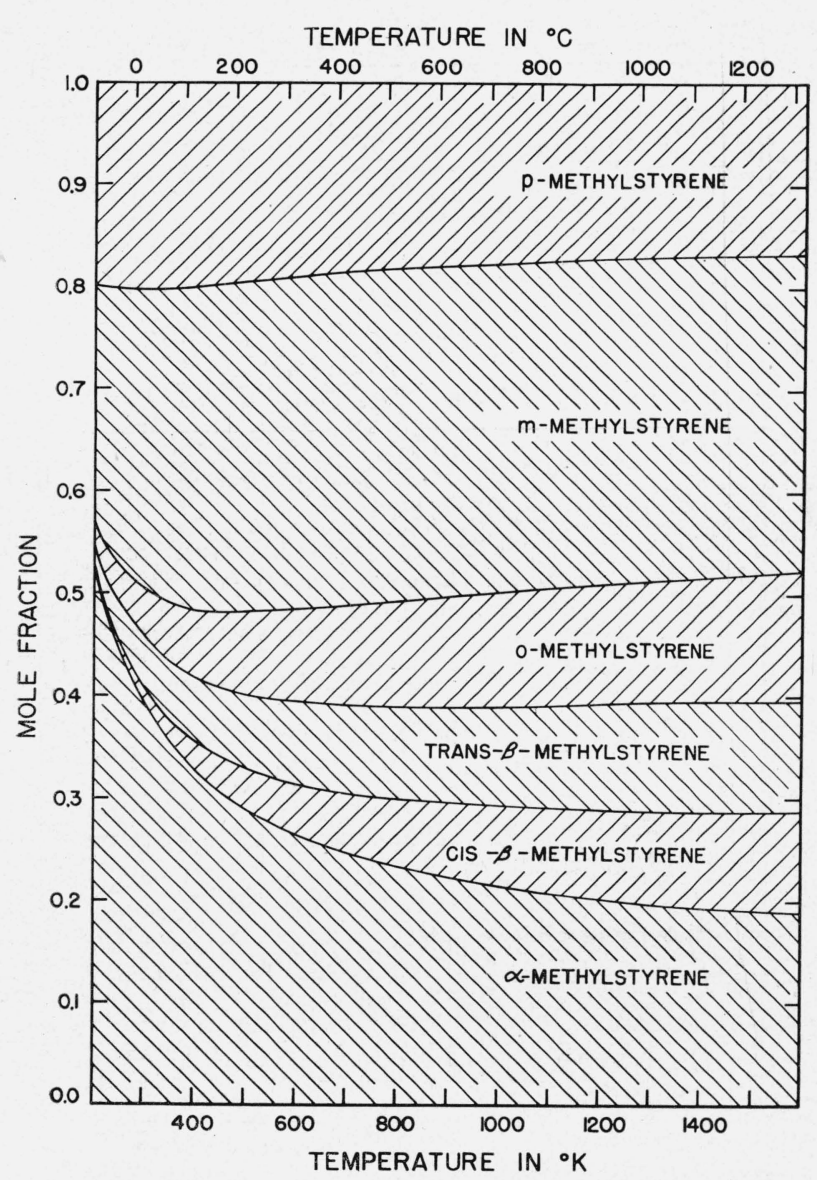

FIGURE 5.-Equilibrium concentrations of the six methylstyrenes.

The scale of ordinates measures the amount in mole fraction, and the scale of abscissas gives the temperature in degrees Kelvin and degrees Centigrade. The vertical width of a band at a given temperature measures the mole fraction of the given isomer present at equilibrium with all of its other isomers in the gaseous state.

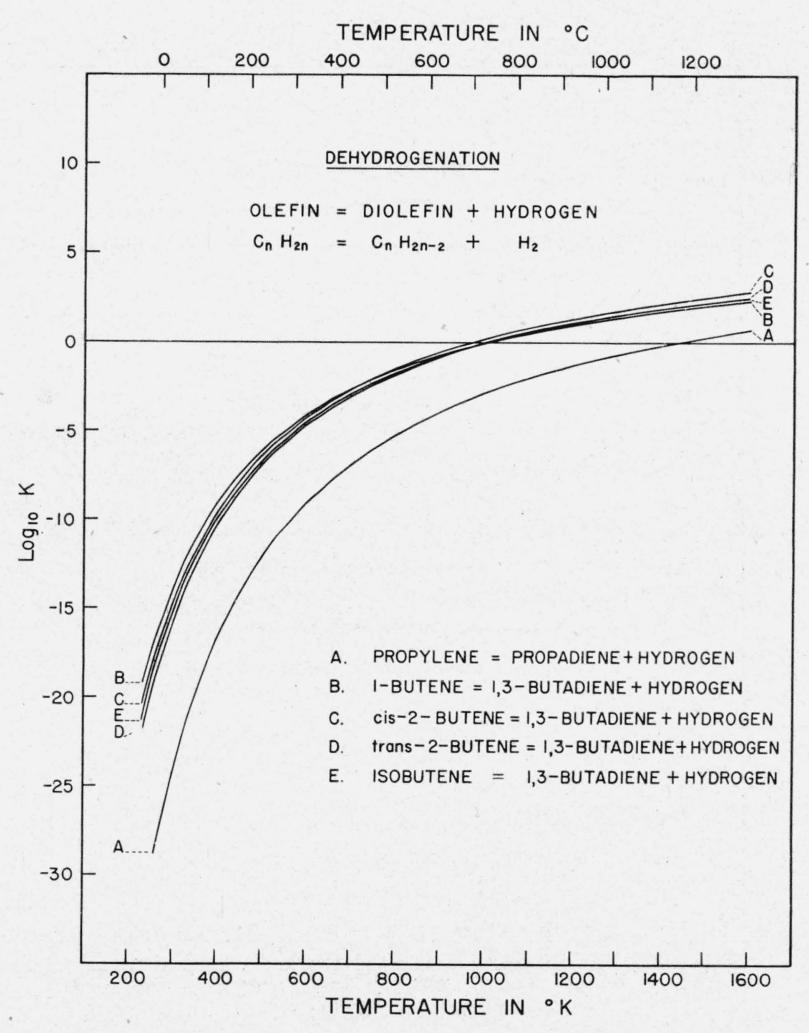

Figure 6. Dehydrogenation of alkylbenzenes to corresponding styrenes.

The scale of ordinates gives the value of the logarithm (to the base 10) of the equilibrium constant for the reaction of dehydrogenating a given alkylbenzene to a corresponding styrene, in the gaseous state. The scale of absiccas gives the temperature in degrees Kelvin. The several curves refer to the following reactions in the gaseous state: A, Ethylbenzene=styrene+hydrogen; $\mathrm{B}$, isopropylbenzene $=\boldsymbol{\alpha} \cdot$ methylstyrene + hydrogen; $\mathrm{C}$, isopropylbenzene $=$ cis- $\beta$-methylstyrene + hydrogen; D, isopropylbenzene $=$ trans $-\beta$-methylstyrene+hydrogen; E, 1-methyl-2-ethylbenzene $=0$-methylstyrene + hydrogen; F, 1-methyl-3-ethylbenzene $=m$-methylstyrene+hydrogen; G, 1-methyl-4ethylbenzene $=p$-methylstyrene + hydrogen . 
In figure 3 are plotted, as a function of temperature, values of the logarithm of the equilibrium constant for the reactions of dehydrogenation of propylene to propadiene and of each of the four butenes to 1,3-butadiene.

In figure 4 are plotted, as a function of temperature, values of the logarithm of the equilibrium constant for the reactions of isomerization of propadiene to propyne, 1,2-butadiene to 1-butyne, 1,2-pentadiene to 1-pentyne, 1,3-butadiene to 2-butyne, cis-1,3-pentadiene to 2-pentyne, and trans-1,3-pentadiene to 2-pentyne.

In figure 5 are plotted, as a function of temperature, for the six methylstyrenes, the amounts, in mole fraction, of each of the isomers present at equilibrium with the other isomers in the gaseous state. The corresponding numerical values are given in table 19.

In figure 6 are plotted, as a function of temperature, values of the logarithm of the equilibrium constant for the reactions of dehydrogenation of ethylbenzene to styrene, isopropylbenzene to $\alpha$-methylstyrene, isopropylbenzene to $\mathrm{cis}-\beta$-methylstyrene, isopropylbenzene to trans- $\beta$-methylstyrene, 1-methyl-2-ethylbenzene to o-methylstyrene, 1-methyl-3-ethylbenzene to m-methylstyrene, and 1-methyl-4-ethylbenzene to $p$-methylstyrene.

Acknowledgment is gratefully made to William Weltner for assistance in the calculations of the thermodynamic functions of the allene-type $\mathrm{C}_{4}$ and $\mathrm{C}_{5}$ diolefins, and also to Helene $\mathrm{G}$. Werner, Rita Braun, and Mary Frances Helm for assistance in other calculations.

\section{References}

[1] Selected values of chemical thermodynamic properties (December 31, 1947). National Bureau of Standards.

[2] Selected values of properties of hydrocarbons. Table $\alpha$. (December 31, 1947). American Petroleum Institute Research Project 44. National Bureau of Standards.

[3] H. W. Thompson and G. P. Harris, Trans. Faraday Soc. 40, 295 (1944).
[4] J. W. Linnett and W. H. Avery, J. Chem. Phys. 6, 686 (1938).

[5] G. Herzberg, Infrared and Raman spectra of polyatomic molecules (D. Van Nostrand Co., New York, N. Y., 1945).

[6] E. H. Eyster, J. Chem. Phys. 6, 580 (1938).

[7] W. S. Gallaway and E. F. Barker, J. Chem. Phys. 10, 88 (1942).

[8] L. Pauling and L. O. Brockway, J. Am. Chem. Soc. 59, 1223 (1937).

[9] G. B. Kistiakowsky and W. W. Rice. J. Chem. Phys. 8, 610 (1940).

[10] G. B. Kistiakowsky, J. P. Lacher, and W. W. Ransom J. Chem. Phys. 8, 970 (1940).

[11] D. A. Frank-Kamenetskii and V. G. Markovich, Acta Physicochim. USSR 17, 308 (1942).

[12] D. A. Frank-Kamenetskii and V. G. Markovich, J. Gen. Chem. USSR 12, 619 (1942).

[13] G. J. Szasz, J. S. McCartney, and D. H. Rank, J. Am. Chem. Soc. 69, 3150 (1947).

[14] J. G. Aston and G. J. Szasz. J. Am. Chem. Soc. 69, 3108 (1947).

[15] K. S. Pitzer and D. W. Scott. J. Am. Chem. Soc. 65, 803 (1943).

[16] J. G. Aston, G. J. Szasz, H. W. Wooley, and F. G. Brickwedde, J. Chem. Phys. 14, 67 (1946).

[17] C. W. Beckett, K. S. Pitzer, and R. Spitzer, J. Am. Chem. Soc. 69, 248 (1947).

[18] K. S. Pitzer. Ind. Eng. Chem. 36, 829 (1944).

[19] J. E. Kilpatrick and K. S. Pitzer, J. Research NBS 3\%, 163 (1946).

[20] W. J. Taylor, D. D. Wagman, M. G. Williams, K. S. Pitzer, and F. D. Rossini, J. Research NBS 37, 95 (1946).

[21] J. E. Kilpatrick, H. G. Werner, C. W. Beckett, K. S. Pitzer, and F. D. Rossini, J. Research NBS 39, 523 (1947).

[22] S. S. Todd, G. D. Oliver, and H. M. Huffman, J. Am. Chem. Soc. 69, 1519 (1947).

[23] H. M. Huffman and D. W. Scott, U. S. Bureau of Mines, Bartlesville, Okla. Private communication.

[24] N. P. Bekkedahl and L. A. Wood, J. Research NBS 19, 55 (1937).

[25] American Petroleum Institute Research Project 44. National Bureau of Standards. Unpublished.

[26] C. W. Beckett and K. S. Pitzer, J. Am. Chem. Soc. 68, 2213 (1946).

[27] E. J. Prosen and F. D. Rossini, J. Research NBS 34, 59 (1945).

[28] E. J. Prosen, F. W. Maron, and F. D. Rossini, National Bureau of Standards. Unpublished.

[29] E. J. Prosen and F. D. Rossini, J. Research NBS 34, 255 (1945). 
[30] E. J. Prosen and F. D. Rossini. J. Research NBS 36, 269 (1946).

[31] E. J. Prosen, W. H. Johnson, and F. D. Rossini, J. Research NBS 36, 455 (1946), RP1714.

[32] E. J. Prosen, W. H. Johnson, and F. D. Rossini, J. Research NBS 3\%, 51 (1946), RP1728.

[33] E. J. Prosen and F. D. Rossini, National Bureau of Standards. Unpublished.

[34] W. H. Johnson, E. J. Prosen, and F. D. Rossini, National Bureau of Standards. Unpublished.
[35] D. D. Wagman, J. E. Kilpatrick, W. J. Taylor, K. S. Pitzer, and F. D. Rossini, J. Research NBS 34, 143 (1945) RP1634.

[36] G. B. Kistiakowsky, J. R. Ruhoff, H. A. Smith, and W. E. Vaughan, J. Am. Chem. Soc. 58, 146 (1936).

[37] M. A. Dolliver, T. L. Gresham, G. B. Kistiakowsky, and W. E. Vaughan, J. Am. Chem. Soc. 59, 831 (1937).

Washington, October 29, 1948. 